\title{
Classically emulated digital quantum simulation for screening and confinement in the Schwinger model with a topological term
}

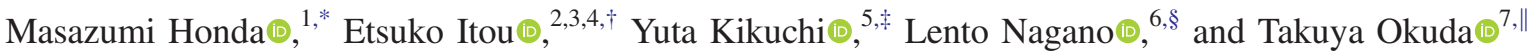 \\ ${ }^{1}$ Center for Gravitational Physics, Yukawa Institute for Theoretical Physics, Kyoto University, \\ Sakyo-ku, Kyoto 606-8502, Japan \\ ${ }^{2}$ Strangeness Nuclear Physics Laboratory, RIKEN Nishina Center, Wako 351-0198, Japan \\ ${ }^{3}$ Keio University, 4-1-1 Hiyoshi, Yokohama, Kanagawa 223-8521, Japan \\ ${ }^{4}$ Research Center for Nuclear Physics (RCNP), Osaka University, Osaka 567-0047, Japan \\ ${ }^{5}$ Department of Physics, Brookhaven National Laboratory, Upton, New York 11973, USA \\ ${ }^{6}$ International Center for Elementary Particle Physics (ICEPP), The University of Tokyo, \\ 7-3-1 Hongo, Bunkyo-ku, Tokyo 113-0033, Japan \\ ${ }^{7}$ Graduate School of Arts and Sciences, University of Tokyo, \\ Komaba, Meguro-ku, Tokyo 153-8902, Japan
}

(Received 20 May 2021; revised 26 October 2021; accepted 22 December 2021; published 7 January 2022)

\begin{abstract}
We perform digital quantum simulation, using a classical simulator, to study screening and confinement in a gauge theory with a topological term, focusing on $(1+1)$-dimensional quantum electrodynamics (Schwinger model) with a theta term. We compute the ground state energy in the presence of probe charges to estimate the potential between them, via adiabatic state preparation. We compare our simulation results and analytical predictions for a finite volume, finding good agreements. In particular our result in the massive case shows a linear behavior for noninteger charges and a nonlinear behavior for integer charges, consistently with the expected confinement (screening) behavior for noninteger (integer) charges.
\end{abstract}

DOI: 10.1103/PhysRevD.105.014504

\section{INTRODUCTION}

A historical milestone of the classical lattice simulation of quantum chromodynamics (QCD) was the derivation of the confinement potential from the first principles [1-3]. The potential energy $V(R)$ between a quark and an antiquark was obtained, via the relation $\langle W(R, T)\rangle \sim$ $\mathrm{e}^{-V(R) T}$ for large $T$, by calculating the expectation value of the rectangular Wilson loop $W(R, T)$ with spatial distance $R$ and temporal separation $T$. The resulting potential $V(R)$ can be fitted well [4] by a function of the form $\sigma R-\alpha / R$ with $\sigma$ and $\alpha$ constants [5]. The linear growth of $V(R)$ for large $R$ is evidence, or the definition, of quark confinement, whose strength is quantified by the string tension $\sigma$.

\footnotetext{
*masazumi.honda@yukawa.kyoto-u.ac.jp

itou@yukawa.kyoto-u.ac.jp

ykikuchi@bnl.gov

\$lento@icepp.s.u-tokyo.ac.jp

takuya@hep1.c.u-tokyo.ac.jp
}

Published by the American Physical Society under the terms of the Creative Commons Attribution 4.0 International license. Further distribution of this work must maintain attribution to the author(s) and the published article's title, journal citation, and DOI. Funded by SCOAP.
The conventional lattice QCD is based on the Lagrangian formalism and the path integral is usually computed by the Markov chain Monte Carlo algorithm, where the Euclidean action $S$ defines the probability $\mathrm{e}^{-S}$ assigned to a field configuration on discretized spacetime. While very successful for real $S$, the conventional lattice simulation becomes practically intractable when $S$ is complex and the integrand is highly oscillatory. This is called the sign problem and arises, for example, when we turn on the topological (theta) term in the action, introduce quark chemical potentials, or consider the real-time dynamics of QCD. These situations occur in many important subjects such as the strong $C P$ problem, neutron stars, the early universe and collider experiments. Various alternative methods have been proposed within the path integral formalism. See, for example, [6].

In digital quantum simulation, where the Hamiltonian formalism is favored and quantum operations over the exponentially large Hilbert space are expected to be efficiently processed, the sign problem tied to the path integral is absent from the beginning. We still need to formulate the theory on a finite lattice and regularize the infinite-dimensional Hilbert space. While the small number of qubits and high error rates severely limit the capability of current quantum computers, it is hoped that quantum hardware with sufficient (supreme) abilities will be built 
in the near (far) future to allow digital simulation of quantum field theories. Before such machines are realized, it is important to develop simulation methods and demonstrate their usefulness. Even now we can use a simulator on a classical computer to perform quantum simulations with a moderate number of qubits (up to 20 or 30 qubits depending on tasks) and without quantum noise.

In this work we perform digital quantum simulation on a classical simulator to study screening and confinement in a gauge theory with a topological term. We focus on $(1+1)$ dimensional quantum electrodynamics (Schwinger model [7]), whose Lagrangian with a theta term [8] is given by ${ }^{1}$

$$
\begin{aligned}
\mathcal{L}_{\mathrm{con}}= & -\frac{1}{4} F_{\mu \nu} F^{\mu \nu}+\frac{g \theta}{4 \pi} \epsilon_{\mu \nu} F^{\mu \nu} \\
& +\mathrm{i} \bar{\psi} \gamma^{\mu}\left(\partial_{\mu}+\mathrm{i} g A_{\mu}\right) \psi-m \bar{\psi} \psi .
\end{aligned}
$$

Here $\psi$ is the Dirac fermion, $g$ the gauge coupling, and $m$ the fermion mass. The model provides a good testing ground for quantum algorithms as it is one of the simplest nontrivial gauge theories that share some features with QCD. With two probe particles of charges $\pm q$, the model is known to exhibit qualitatively different behaviors depending on the values of $q$ and $m$ (for $\theta=0$ ): with $m=0$ the two probe charges are screened for any value of $q$ [9], while with $m \neq 0$ they are confined for noninteger $q$ and screened for integer $q$ [8].

To study the screening versus confinement problem, we compute the ground state energy in the presence of probe charges and estimate the potential between them. We put the Schwinger model on a lattice and introduce two probe charges $\pm q$ using a position-dependent theta angle. Then we adiabatically prepare the ground state of the Hamiltonian and compute its energy as a function of a distance between the probes. ${ }^{2}$ This is an analog of the quark-antiquark potential while our computational scheme should be contrasted with the one in the conventional lattice QCD. Our simulation results in the massless case agree well with the analytic results for a finite volume in the continuum limit. In the massive case, our result shows a linear behavior for noninteger $q$ just like the confining potential in the infinite-volume limit does. The present work envisions what the investigation will look like when one studies screening and confinement on a real quantum computer in the future. We believe that our work will serve as a guide for the simulation of many-body physics in the noisy intermediate-scale quantum (NISQ)

\footnotetext{
${ }^{1}$ Our convention: $\eta_{\mu \nu}=\operatorname{diag}(1,-1), \epsilon_{01}=1, F_{\mu \nu}=\partial_{\mu} A_{\nu}-$ $\partial_{\nu} A_{\mu}, \gamma^{0}=\sigma_{z}, \gamma^{1}=\mathrm{i} \sigma_{y}, \gamma^{5}=\sigma_{x}, \bar{\psi}=\psi^{\dagger} \gamma^{0}$.

${ }^{2}$ One can use an adiabatic process to prepare the ground state accurately for finite-gap systems by taking a sufficiently large adiabatic time. In the fault-tolerant era it is anticipated to be more useful than variational methods.
}

[10]/early fault-tolerant era, when the number of qubits will be limited. ${ }^{3}$

\section{QUBIT DESCRIPTION OF THE SCHWINGER MODEL}

We begin by rewriting the Hamiltonian of the Schwinger model in terms of spin operators acting on qubits, following $[11,71,72]$ but with a theta angle [16]. The Hamiltonian of the continuum theory in the gauge $A_{0}=0$ is $^{4}$

$$
\int d x\left[\frac{1}{2}\left(\Pi-\frac{g \theta}{2 \pi}\right)^{2}-\mathrm{i} \bar{\psi} \gamma^{1}\left(\partial_{1}+\mathrm{i} g A_{1}\right) \psi+m \bar{\psi} \psi\right],
$$

where $\Pi=\partial_{0} A^{1}+g \theta / 2 \pi$ is the canonical momentum conjugate to $A^{1}$ and physical states are subject to the Gauss law $\partial_{1} \Pi+g \psi^{\dagger} \psi=0$.

We regularize the theory by putting it on a onedimensional spatial lattice with $N$ sites, lattice spacing $a$ and open boundaries. We replace the Dirac fermion $\psi(x)=$ $\left(\psi_{u}(x), \psi_{d}(x)\right)^{\top}$ by a pair of neighboring staggered fermions [73] according to

$$
\frac{\chi_{n}}{\sqrt{a}} \leftrightarrow \begin{cases}\psi_{u}(x) & n: \text { even }, \\ \psi_{d}(x) & n: \text { odd },\end{cases}
$$

where $n=0, \ldots, N-1$ label the lattice sites $x=n a$. The lattice counterparts of the gauge field operators are $U_{n}$ $\left(\leftrightarrow \mathrm{e}^{-\mathrm{i} a g A^{1}(x)}\right)$ and $L_{n}(\leftrightarrow-\Pi(x) / g)$ such that $U_{n}^{\dagger}=U_{n}^{-1}$, $L_{n}^{\dagger}=L_{n}$, defined on the link between the $n$th and $(n+1)$ th sites. These operators satisfy the canonical commutation relations

$$
\left\{\chi_{n}^{\dagger}, \chi_{m}\right\}=\delta_{m n}, \quad\left\{\chi_{n}, \chi_{m}\right\}=0, \quad\left[U_{n}, L_{m}\right]=\delta_{m n} U_{n} .
$$

Let us introduce the probe charges $+q$ and $-q$ on the $\hat{\ell}_{0}$ th and $\left(\hat{\ell}_{0}+\hat{\ell}\right)$ th sites, respectively. We realize this by making the theta angle position dependent as

$$
\vartheta_{n}= \begin{cases}2 \pi q+\theta_{0}, & \hat{\ell}_{0} \leq n<\hat{\ell}_{0}+\hat{\ell}, \\ \theta_{0}, & \text { otherwise. }\end{cases}
$$

We place the probe charges equally separated from the center of the lattice by setting $\hat{\ell}_{0}=(N-1-\hat{\ell}) / 2$. The lattice Hamiltonian with the probe charges is

\footnotetext{
${ }^{3}$ Previous works that study digital quantum simulation of the Schwinger model include [11-19]. See also [20,21] for analog simulations of the Schwinger model, [22-54] for digital simulations of other quantum field theories, and [55-67] for their analog simulations. Algorithms specific to the simulation of gauge theories were developed in [48,68-70].

${ }^{4}$ In contrast to [16], we do not absorb the theta angle by a chiral rotation of the mass term.
} 


$$
\begin{aligned}
H= & J \sum_{n=0}^{N-2}\left(L_{n}+\frac{\vartheta_{n}}{2 \pi}\right)^{2}-\mathrm{i} w \sum_{n=0}^{N-2}\left(\chi_{n}^{\dagger} U_{n} \chi_{n+1}-\chi_{n+1}^{\dagger} U_{n}^{\dagger} \chi_{n}\right) \\
& +m \sum_{n=0}^{N-1}(-1)^{n} \chi_{n}^{\dagger} \chi_{n},
\end{aligned}
$$

where $w=1 /(2 a)$ and $J=g^{2} a / 2$. The physical states must satisfy the lattice version of the Gauss law

$$
L_{n}-L_{n-1}=\chi_{n}^{\dagger} \chi_{n}-\frac{1-(-1)^{n}}{2} .
$$

We solve this with the boundary condition $L_{-1}=0$ and fix the gauge $U_{n}=1$ to eliminate $\left(L_{n}, U_{n}\right)$.

To rewrite the theory in terms of a spin system, we apply the Jordan-Wigner transformation [74]

$$
\chi_{n}=\frac{X_{n}-\mathrm{i} Y_{n}}{2} \prod_{i=0}^{n-1}\left(-\mathrm{i} Z_{i}\right)
$$

where $\left(X_{n}, Y_{n}, Z_{n}\right)$ denote the Pauli matrices $\left(\sigma_{x}, \sigma_{y}, \sigma_{z}\right)$ acting on the qubit on site $n$, then the lattice Hamiltonian becomes [73]

$$
\begin{aligned}
H= & J \sum_{n=0}^{N-2}\left[\sum_{i=0}^{n} \frac{Z_{i}+(-1)^{i}}{2}+\frac{\vartheta_{n}}{2 \pi}\right]^{2} \\
& +\frac{w}{2} \sum_{n=0}^{N-2}\left[X_{n} X_{n+1}+Y_{n} Y_{n+1}\right]+\frac{m}{2} \sum_{n=0}^{N-1}(-1)^{n} Z_{n},
\end{aligned}
$$

up to an irrelevant constant. In this form, it is manifest that we can directly apply quantum algorithms in qubit form to the lattice Schwinger model.

\section{SIMULATION PROTOCOL}

Our main target is the energy

$$
E\left(\theta_{0}, q, \ell\right):=\langle\mathrm{GS}|H| \mathrm{GS}\rangle=\langle H\rangle
$$

of the ground state $|\mathrm{GS}\rangle$ in the presence of probes, where the parameters $\left(\theta_{0}, q, \ell=\hat{\ell} a\right)$ enter the setup as described above. We will refer to $V_{f}\left(\theta_{0}, q, \ell\right):=E\left(\theta_{0}, q, \ell\right)-$ $E(0,0,0)$ as the potential. ${ }^{5}$ We use the adiabatic state preparation and the Suzuki-Trotter decomposition to obtain an approximation to the ground state $|\mathrm{GS}\rangle$.

Set $H_{0}:=\left.H\right|_{w=0, \theta_{0}=0, q=0, m=m_{0}}$ for some $m_{0}>0$. Its ground state is $\left|\mathrm{GS}_{0}\right\rangle=|1010 \ldots\rangle$ with $Z|0\rangle=+|0\rangle$ and $Z|1\rangle=-|1\rangle$, and can be constructed easily. Fix $T>0$ and choose a one-parameter family of slowly varying Hamiltonians $H_{A}(t)(0 \leq t \leq T)$ such that

$$
H_{A}(0)=H_{0}, \quad H_{A}(T)=H .
$$

By the adiabatic theorem, if $H_{A}(t)$ has a unique gapped ground state for any $t$, then the exact ground state $|\mathrm{GS}\rangle$ is given by

$$
|\mathrm{GS}\rangle=\lim _{T \rightarrow \infty} \mathcal{T} \exp \left(-\mathrm{i} \int_{0}^{T} d t H_{A}(t)\right)\left|\mathrm{GS}_{0}\right\rangle,
$$

where $\mathcal{T}$ denotes time ordering. In practice, we take $T$ to be sufficiently large but finite, which induces a systematic error in the preparation of the ground state (see, e.g., [75,76] and Appendix A).

To apply the Suzuki-Trotter decomposition, we decompose the Hamiltonian as

$$
H=H_{X Y}^{(0)}+H_{X Y}^{(1)}+H_{Z}+C
$$

Each term is given as

$$
\begin{aligned}
H_{X Y}^{(0)}= & \frac{w}{2} \sum_{m=0}^{\frac{N-3}{2}}\left(X_{2 m} X_{2 m+1}+Y_{2 m} Y_{2 m+1}\right), \quad H_{X Y}^{(1)}=\frac{w}{2} \sum_{m=1}^{\frac{N-1}{2}}\left(X_{2 m-1} X_{2 m}+Y_{2 m-1} Y_{2 m}\right), \\
H_{Z}= & \frac{J}{2} \sum_{n=0}^{N-3} \sum_{m=n+1}^{N-2}(N-m-1) Z_{n} Z_{m}+\frac{J}{2} \sum_{n=0}^{N-2} \frac{1+(-1)^{n}}{2} \sum_{i=0}^{n} Z_{i}+q J \sum_{m=0}^{\hat{e}_{0}+\hat{\ell}-1}\left(\hat{\ell}_{0}+\hat{\ell}-m\right) Z_{m} \\
& -q J \sum_{m=0}^{\hat{\ell}_{0}-1}\left(\hat{\ell}_{0}-m\right) Z_{m}+\frac{\theta_{0}}{2 \pi} J \sum_{m=0}^{N-2}(N-m-1) Z_{m}+\frac{m}{2} \sum_{n=0}^{N-1}(-1)^{n} Z_{n}, \\
C= & \frac{q J}{2}\left(\hat{\ell}+(-1)^{\hat{\ell}_{0}} \frac{1-(-1)^{\hat{\ell}}}{2}\right)+q\left(q+\frac{\theta_{0}}{\pi}\right) J \hat{\ell}+\frac{\theta_{0} J}{4 \pi}\left(N-1+\frac{1+(-1)^{N}}{2}\right)+\left(\frac{\theta_{0}}{2 \pi}\right)^{2} J(N-1)
\end{aligned}
$$

\footnotetext{
${ }^{5}$ The $\ell$-dependent part is free from UV divergence and therefore the potential has a well-defined continuum limit.
} 
for odd $N$, on which we focus in this work. ${ }^{6}$ Here $C$ is a classical number where we have kept only the terms that depend on $\theta_{0}, q, \hat{\ell}$. All the terms commute within each of $H_{X Y}^{(0)}, H_{X Y}^{(1)}$, and $H_{Z}$.

Let $H_{X Y, s}^{(0)}, H_{X Y, s}^{(1)}$, and $H_{Z, s}$ denote the modifications of the corresponding operators in (12) by the replacements

$$
\begin{aligned}
& w \rightarrow w \frac{s \delta t}{T}, \quad \theta_{0} \rightarrow \theta_{0} \frac{s \delta t}{T}, \quad q \rightarrow q \frac{s \delta t}{T}, \\
& m \rightarrow m_{0}\left(1-\frac{s \delta t}{T}\right)+m \frac{s \delta t}{T} .
\end{aligned}
$$

We approximate (10) by the second-order Suzuki-Trotter decomposition as

$$
\begin{aligned}
\left|\mathrm{GS}_{A}\right\rangle:= & \prod_{s=1}^{M}\left(\mathrm{e}^{-\mathrm{i} H_{X Y, S}^{(0)} \mathrm{\delta t}} \mathrm{e}^{-\mathrm{i} H_{X Y, S}^{(1)}} \mathrm{e}^{-\mathrm{i} H_{Z, S} \delta t}\right. \\
& \left.\times \mathrm{e}^{-\mathrm{i} H_{X Y, s \frac{\delta}{2}}^{(1)}} \mathrm{e}^{-\mathrm{i} H_{X Y, S \frac{\delta}{2}}^{(0)}}\right)\left|\mathrm{GS}_{0}\right\rangle
\end{aligned}
$$

where $M:=T / \delta t$ and the product is ordered from right to left with increasing $s$. For fixed $T$, this approximation results in the error of $\mathcal{O}\left(\delta t^{2}\right)$ for the whole operator [77,78]. Then we compute $\left\langle\mathrm{GS}_{A}|H| \mathrm{GS}_{A}\right\rangle$, which approximates the ground state energy. This involves separate measurements of the mutually noncommuting terms in the Hamiltonian (11): $H_{X Y}^{(0)}, H_{X Y}^{(1)}$ and $H_{Z}$. We denote the number of times the circuit for each term is executed by $n_{\text {shots }}$, which determines the statistical uncertainties in the simulation. Details on the above protocol are explained in Appendix B.

Let us comment on a property of the state $\left|\mathrm{GS}_{A}\right\rangle$. The time evolution in (14) preserves the $\mathrm{U}(1)$ symmetry generated by $Q:=\sum_{n=0}^{N-1} Z_{n}$ and the state $\left|\mathrm{GS}_{A}\right\rangle$ approximates the ground state within the $Q=-1$ sector. In principle, there may be states with lower energies with different charges. See Appendixes A and C for details.

\section{SIMULATION RESULTS}

We present the results of our quantum simulations on a classical simulator (QasmSimulator in IBM's open source SDK Qiskit). In this section we fix $a=0.4 g^{-1}$, $\delta t=0.3 g^{-1}, T=99 g^{-1}$ and $m_{0}=0.5 g$. Therefore the physical volume $L=a(N-1)$ is simply specified by $N$. In Appendix A we show that the systematic errors are comparable to the statistical uncertainties in the whole parameter region we study while the systematic errors become larger when we expect a larger effect of a phase transition, ${ }^{7}$ i.e., for larger $\theta_{0}, m, q$, and $\ell$. Analytical results

\footnotetext{
${ }^{6}$ In these expressions, only $X_{n}, Y_{n}, Z_{n}$ are quantum operators and all the other quantities are c-numbers.

${ }^{7}$ The continuum Schwinger model in the infinite volume undergoes a first-order phase transition at $\theta_{0}=\pi$ and for $m$ larger than a critical mass $m_{c}$ [79], with value $m_{c} / g=0.3335(2)$ [80].
}

in a finite volume compared here are derived in Appendix D 2.

We begin with the $\theta_{0}=0$ case. In Fig. 1 , we show the simulation results for the potential $V_{f}$ with $q=1$ as a representative of the integer $q$ case where the theory is known to exhibit a screening behavior. For $m=0$ (left panel), we compare the simulation results with the potential in the infinite volume [81]

$$
V^{(0)}(\ell)=\frac{\sqrt{\pi} q^{2} g}{2}\left(1-\mathrm{e}^{-\frac{g \ell}{\sqrt{\pi}}}\right)
$$

and the one in a finite volume

$$
V_{f}^{(0)}(\ell)=\frac{\sqrt{\pi} q^{2} g}{2} \frac{\left(1-\mathrm{e}^{-\frac{g \ell}{\sqrt{\pi}}}\right)\left(1+\mathrm{e}^{-\frac{g(L-\ell)}{\sqrt{\pi}}}\right)}{1+\mathrm{e}^{-\frac{g L}{\sqrt{\pi}}}} .
$$

For $m / g=0.2$ (right panel), the curves represent $V_{f}^{(0)}+V_{f}^{(1)}$, where $V_{f}^{(1)}$ is the $\mathcal{O}(m)$ mass perturbation given in (D10). The simulation results agree rather well with the analytical predictions with the same volumes. We cannot see a clear plateau although the results for a larger volume $(N=21)$ have a more slowly varying region in which the values of the potential are close to that of the plateau for a very large volume $(N=101, L g=40) .{ }^{8}$ The value of the plateau for a large volume depends on $m$ and this is a result of nontrivial dynamics. The absence of a clear plateau in the simulation is reasonable because the simulation setup does not have a parameter region that satisfies the condition $1 \ll g \ell \ll g L$ necessary to have a plateau. The small discrepancy between the simulation data and the corresponding curves indicates a correction due to finite $a$ and/or systematic errors.

In the left panel of Fig. 2, we depict the simulation results for $q=0.25$ as a representative of noninteger charge where the confinement (screening) behavior is expected for massive (massless) case. We first observe a qualitative difference between the simulation data for the massive and massless cases: the $m / g=0.2$ case exhibits an almost linear behavior in the region $g \ell \gtrsim 3$ while the massless case does not. This is in contrast to the integer $q$ case demonstrated in Fig. 1, where the screening behavior is expected for any $m$. Thus our simulation results are consistent with the above expectations although a larger volume is needed for unambiguous demonstrations. More quantitatively, we compare the simulation results with the analytical results on a finite volume. For $m=0$ the simulation results agree well with the analytical prediction $V_{f}^{(0)}(\ell)$ as can be seen in the left panel of Fig. 1. The data for $m / g=0.2$ has moderate discrepancies from the $\mathcal{O}(m)$

\footnotetext{
${ }^{8}$ With $L g=12(N=31)$, the function $V_{f}^{(0)}+V_{f}^{(1)}$ exhibits a plateau of the height within 3.5 percent of the height for $L g=40$ $(N=101)$.
} 

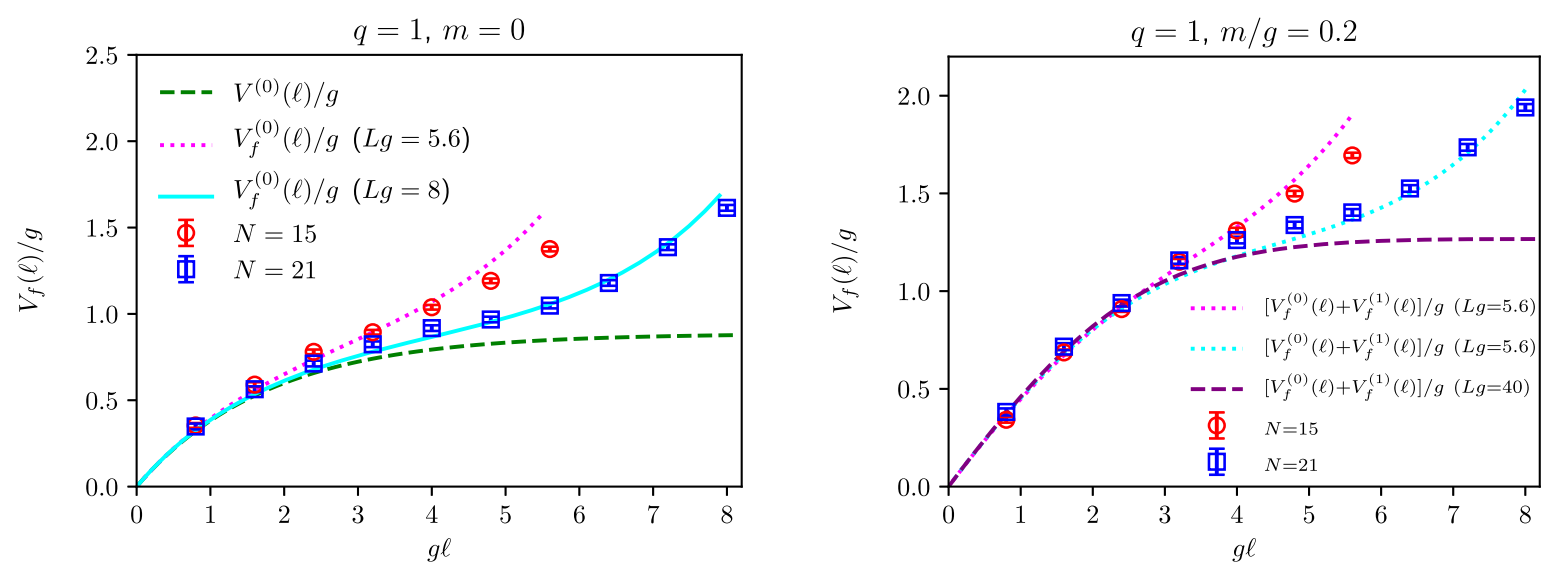

FIG. 1. Results for $V_{f}(\ell)$ with $n_{\text {shots }}=10^{5}$. The statistical uncertainties are smaller than the markers. The curves (in colors similar to and lighter than the data markers) represent $V_{f}^{(0)}(\ell)$ on the left and $V_{f}^{(0)}(\ell)+V_{f}^{(1)}(\ell)$ on the right with the corresponding volumes. The dashed curve (purple) on the right panel depicts $V_{f}^{(0)}(\ell)+V_{f}^{(1)}(\ell)$ for $N=101, L g=40$.
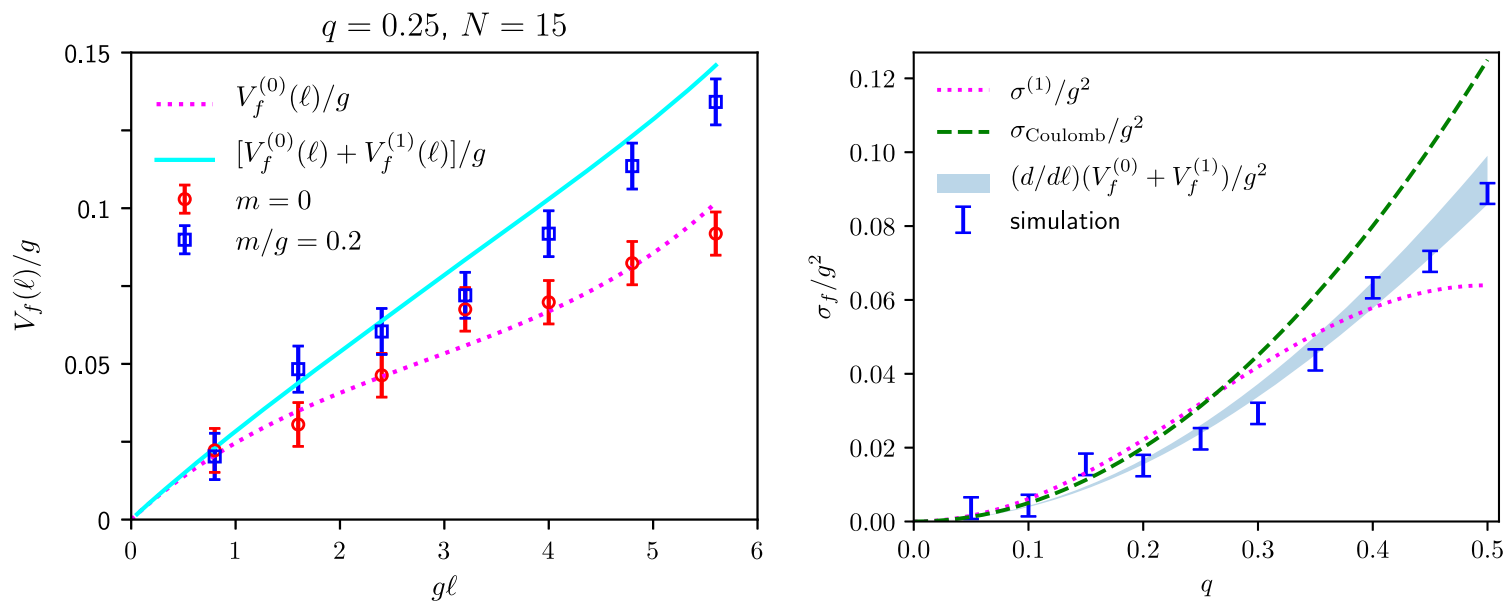

FIG. 2. Left: results for $V_{f}(\ell)$ with $n_{\text {shots }}=4 \times 10^{5}$. The error bars represent the statistical uncertainties. The solid (cyan) and dotted (magenta) curves represent $V_{f}^{(0)}$ and $V_{f}^{(0)}+V_{f}^{(1)}$, respectively. Right: the finite-volume analog $\sigma_{f}$ of the string tension for $m / g=0.2$. The error bars represent the standard uncertainties from the fit. The dashed (green) and dotted (magenta) curves represent $\sigma_{\mathrm{Coulomb}} / g^{2}$ and $\sigma^{(1)} / g^{2}$, respectively. The band region represents the finite-volume counterpart of $\sigma^{(1)} / g^{2}$.

analytical prediction $V_{f}^{(0)}(\ell)+V_{f}^{(1)}(\ell)$. In Appendix E, we study the mass dependence of the potential and argue that their origin is likely due to the inadequacy of the $\mathcal{O}(m)$ approximation.

In the right panel of Fig. 2, we plot of the string tension a finite- $L\left(\sigma_{f}\right)$ for various values of $q$, where $\sigma_{f}$ is defined as the slope given by a linear fit for the energy $E(\ell)$ as a function of $\ell$. Specifically the linear fit has been done by least squares weighted by the statistical uncertainties in the region $2.4 \leq g \ell \leq 4.8(\hat{\ell}=6,8,10,12) .{ }^{9}$ We first observe that our simulation data clearly deviate from the string

\footnotetext{
${ }^{9}$ To estimate the true string tension, we should take a larger volume and choose a fit range away from $g \ell=0$ and $g L$.
}

tension $\sigma_{\text {Coulomb }}=q^{2} g^{2} / 2$ of the classical Coulomb potential (corresponding to infinite mass), implying that our simulation result exhibits confinement by nontrivial dynamics. More quantitatively, we again compare the result with the $\mathcal{O}(m)$ analytical results both on the infinite and finite volumes. The string tension in the infinite volume is

$$
\sigma^{(1)}(q)=\frac{\mathrm{e}^{\gamma} m g}{2 \pi^{3 / 2}}[1-\cos (2 \pi q)]
$$

with $\gamma$ being the Euler constant [9]. We depict its finitevolume counterpart as the band region surrounded by the maximum and minimum of the derivative $(d / d \ell)\left(V_{f}^{(0)}+\right.$ $\left.V_{f}^{(1)}\right) / g^{2}$ in the range $2.4 \leq g \ell \leq 4.8$ used for the linear 


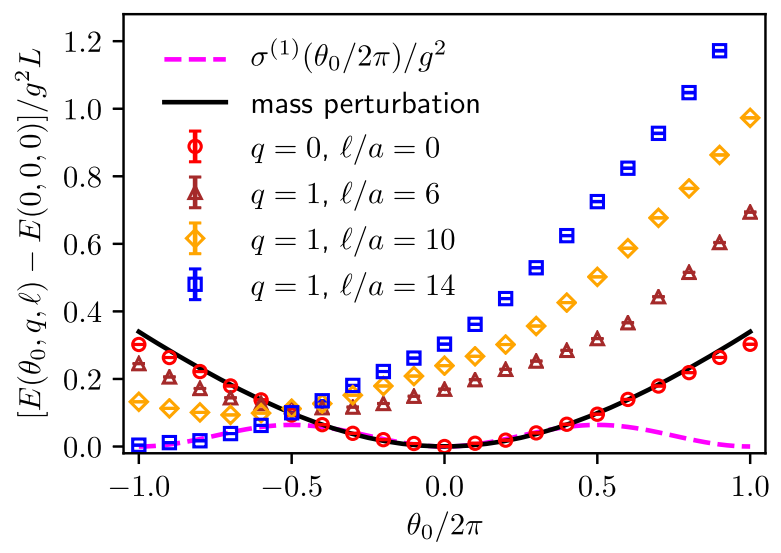

FIG. 3. Results for $\left[E\left(\theta_{0}, q, \ell\right)-E(0,0,0)\right] / g^{2} L$ with $N=$ $15, m / g=0.2, a g=0.4$ and $n_{\text {shots }}=10^{5}$. The statistical uncertainties are much smaller than the markers. The solid (black) and dashed (magenta) curves represent the results of the mass perturbation theory with $q=0$ and $\sigma^{(1)}\left(\theta_{0} / 2 \pi\right) / g^{2}$, respectively.

fit. ${ }^{10}$ While the simulation result is roughly consistent with both, it is somewhat closer to the finite-volume result for $q \gtrsim 0.4$.

Let us turn to nonzero $\theta_{0}$, which is inaccessible by the conventional Monte Carlo approach unless $\theta_{0}$ is small. In Fig. 3, we plot the simulation results for $\left[E\left(\theta_{0}, q, \ell\right)-\right.$ $E(0,0,0)] / g^{2} L$ against $\theta_{0}$ for various values of $\ell$. One can read off the $\ell$-dependence of the potential from the simulation data at fixed $\theta_{0}$. The simulation result for $q=$ 0 agrees well with the $\mathcal{O}(m)$ analytical result on a finite volume given explicitly by (D5) and (D8). For $\theta_{0}$ close to zero, the analytic formula $\sigma^{(1)}\left(\theta_{0} / 2 \pi\right)$ viewed as the energy density is also roughly consistent with the simulation result.

Note that the simulation results do not show the periodicity $\theta_{0} \sim \theta_{0}+2 \pi$. This is expected for the open boundary condition because the theory with the theta angle $\theta_{0}+2 \pi k(k \in \mathbb{Z})$ is equivalent to the one with $\theta_{0}$ in the presence of extra probe charges $\pm k$ on the boundaries. Indeed in Fig. 3, the blue squares in the range $-1 \leq$ $\theta_{0} / 2 \pi \leq 0$ coincide with the red circles in the range $0 \leq$ $\theta_{0} / 2 \pi \leq 1$ up to a horizontal shift.

\section{DISCUSSION}

We used the classical simulator to test and demonstrate our protocol. Let us estimate the resources needed to implement the algorithm on real quantum devices expected to be available in the near and far futures. (For more details, see Appendix B 3.) In the near future, only NISQ devices will be available. On such devices, single-qubit gates will be implemented more accurately and with higher speed

\footnotetext{
${ }^{10}$ In Appendix D 3 we show that $N=35$ is enough to have good agreement between $(d / d \ell)\left(V_{f}^{(0)}+V_{f}^{(1)}\right) / g^{2}$ and $\sigma^{(1)}$ for $m / g=0.2$ and $g a=0.4$.
}

than two-qubit gates, which will be the dominant cause of the quantum error. In our algorithm the only two-qubit gates are the CNOT gates, and we need $\mathcal{O}\left(M N^{2}\right)$ of them to perform the discrete time evolution of $M$ Suzuki-Trotter steps in (14) for $N$ lattice sites. Thus our simulation with $N=15$ and $M=330$ would require roughly a hundred thousand CNOT gates. This makes the NISQ implementation of our simulation implausible.

In the far future, quantum devices with many qubits and less noise will be able to implement quantum error correction. In most approaches to fault-tolerance [82], the $T$-gate will consume the most computational cost among the Clifford $+T$ universal gate set. Our algorithm demands $\mathcal{O}\left(N^{2} \log \left(N^{2} / \delta\right)\right) \quad T$-gates to implement one Suzuki-Trotter step within an accuracy $\delta$.

We have introduced two probe charges using a positiondependent theta angle. In an Abelian gauge theory, this method is equivalent to the one based on a change of the Gauss law [83]. In non-Abelian gauge theories, an approach similar to ours is to introduce probes as heavy fermions and measure the energy [84,85]. In higher dimensions the quark-antiquark potential can also be estimated by Wilson loops along spatial directions as in the conventional lattice QCD. The approach based on the inclusion of probes as part of the Hamiltonian is in contrast with the conventional method based on Wilson loops, where one suffers from a large signal-to-noise ratio in extracting the string tension, although several smearing techniques have been applied to reduce statistical uncertainties. It would be important to implement this approach in quantum simulation.

\section{ACKNOWLEDGMENTS}

We would like to thank Mitsuhiro Kato and Yuya Tanizaki for useful conversations. We also thank Y. Tanizaki for pointing out a mistake in an analytic computation in an earlier version. M. H. is partially supported by MEXT Q-LEAP. The work of E. I. is supported by JSPS KAKENHI with Grants No. 19K03875 and No. JP18H05407, and by the HPCI-JHPCN System Research Project (Project ID: jh210016). Y. K. is supported by the U.S. Department of Energy, Office of Science, National Quantum Information Science Research Centers, Co-design Center for Quantum Advantage, under the Contract No. DE-SC0012704. Discussions during the Yukawa Institute for Theoretical Physics (YITP) workshop YITP-W-20-17 on "Quantum computing for quantum field theories" were useful for completing this work.

\section{APPENDIX A: SYSTEMATIC ERRORS}

The expectation value of an operator $\mathcal{O}$ in the true ground state $|\mathrm{GS}\rangle$ is defined as

$$
\langle\mathcal{O}\rangle=\langle\mathrm{GS}|\mathcal{O}| \mathrm{GS}\rangle
$$


TABLE I. Numerical results for $E(\ell) / g$ obtained by exact diagonalization and quantum simulation without statistical uncertainties for $N=15, a g=0.4, m=0, q=0.5$, and $\theta_{0}=0$. Systematic errors $\delta_{\text {sys }} E=\delta_{\text {sys }}\langle H\rangle$ and statistical uncertainties $\delta_{\text {stat }} E$ for $n_{\text {shots }}=10^{5}$ are also shown.

\begin{tabular}{lcccc}
\hline \hline$g \ell$ & Exact diagonalization & Quantum simulation & Systematic error & Statistical uncertainty \\
\hline 0.0 & -16.2954 & -16.2897 & $5.7 \mathrm{E}-003(0.03 \%)$ & $1.00 \mathrm{E}-002(0.06 \%)$ \\
0.8 & -16.2128 & -16.2072 & $5.6 \mathrm{E}-003(0.03 \%)$ & $1.00 \mathrm{E}-002(0.06 \%)$ \\
1.6 & -16.1506 & -16.1448 & $5.8 \mathrm{E}-003(0.04 \%)$ & $0.99 \mathrm{E}-002(0.06 \%)$ \\
2.4 & -16.1057 & -16.0986 & $7.1 \mathrm{E}-003(0.04 \%)$ & $0.99 \mathrm{E}-002(0.06 \%)$ \\
3.2 & -16.0590 & -16.0533 & $5.8 \mathrm{E}-003(0.04 \%)$ & $0.98 \mathrm{E}-002(0.06 \%)$ \\
4.0 & -16.0255 & -16.0198 & $5.8 \mathrm{E}-003(0.04 \%)$ & $0.97 \mathrm{E}-002(0.06 \%)$ \\
4.8 & -15.9692 & -15.9619 & $7.3 \mathrm{E}-003(0.05 \%)$ & $0.97 \mathrm{E}-002(0.06 \%)$ \\
5.6 & -15.9248 & -15.9172 & $7.6 \mathrm{E}-003(0.05 \%)$ & $0.97 \mathrm{E}-002(0.06 \%)$ \\
\hline \hline
\end{tabular}

TABLE II. Numerical results for $E(\ell) / g$ obtained for $N=15, a g=0.4, m / g=0.2, q=0.5$, and $\theta_{0}=0$. Statistical uncertainties for $n_{\text {shots }}=10^{5}$ are also shown.

\begin{tabular}{lcccc}
\hline \hline$g \ell$ & Exact diagonalization & Quantum simulation & Systematic error & Statistical uncertainty \\
\hline 0.0 & -16.6458 & -16.6347 & $1.10 \mathrm{E}-002(0.07 \%)$ & $1.05 \mathrm{E}-002(0.06 \%)$ \\
0.8 & -16.5562 & -16.5757 & $1.96 \mathrm{E}-002(0.12 \%)$ & $1.04 \mathrm{E}-002(0.06 \%)$ \\
1.6 & -16.4770 & -16.4710 & $0.60 \mathrm{E}-002(0.04 \%)$ & $1.04 \mathrm{E}-002(0.06 \%)$ \\
2.4 & -16.4052 & -16.4080 & $0.28 \mathrm{E}-002(0.02 \%)$ & $1.03 \mathrm{E}-002(0.06 \%)$ \\
3.2 & -16.3327 & -16.3030 & $2.97 \mathrm{E}-002(0.18 \%)$ & $1.00 \mathrm{E}-002(0.06 \%)$ \\
4.0 & -16.2669 & -16.2532 & $1.37 \mathrm{E}-002(0.08 \%)$ & $1.00 \mathrm{E}-002(0.06 \%)$ \\
4.8 & -16.1905 & -16.1692 & $2.13 \mathrm{E}-002(0.13 \%)$ & $0.98 \mathrm{E}-002(0.06 \%)$ \\
5.6 & -16.1228 & -16.0990 & $2.38 \mathrm{E}-002(0.15 \%)$ & $0.99 \mathrm{E}-002(0.06 \%)$ \\
\hline \hline
\end{tabular}

In our simulation we calculate

$$
\langle\mathcal{O}\rangle_{A}=\left\langle\mathrm{GS}_{A}|\mathcal{O}| \mathrm{GS}_{A}\right\rangle
$$

where $\left|\mathrm{GS}_{A}\right\rangle$ is the approximate ground state defined in (14). Here we study the systematic error

$$
\delta_{\text {sys }}\langle\mathcal{O}\rangle:=\langle\mathcal{O}\rangle-\langle\mathcal{O}\rangle_{A},
$$

where $\langle\mathcal{O}\rangle$ and $\langle\mathcal{O}\rangle_{A}$ are calculated by exact diagonalization and quantum simulation without statistical uncertainties, ${ }^{11}$ respectively. For exact diagonalization we use the Python package QuSpin. For quantum simulation without statistical uncertainties we use the "snapshot" functionality of Qiskit.

Besides the physical parameters of the lattice Schwinger model, adiabatic preparation requires three extra parameters: the initial mass $m_{0}$, the Trotter time step $\delta t$, and the adiabatic time $T$. As in Section IV we fix them to $\delta t=0.3 g^{-1}, T=99 g^{-1}$ and take $m_{0} / g \approx 0.5 .^{12}$

\footnotetext{
${ }^{11}$ This enables us to obtain the actual values of systematic errors rather than estimate systematic uncertainties.

${ }^{12}$ We fix $m_{0} / g=0.5$ for all the simulations in this paper except for the right panel of Fig. $5\left(m_{0} / g=0.55\right)$ and the left panel of Fig. $6\left(m_{0} / g=0.7\right)$.
}

From the numerical results shown in Table I for $m=0$, we see that the systematic errors are smaller than the statistical uncertainties for $n_{\text {shots }}=10^{5}$ estimated by (B12). Table II shows numerical results for the particular value $m / g=0.2$ of mass. Systematic errors are larger than statistical uncertainties, though they are still of the same order of magnitude. We also see the tendency that the systematic error gets larger for larger $g \ell^{13}$

There is a known bound on the adiabatic error (see, for example, $[75,76,86])$. Let us define adiabatic error $\epsilon$ as

$$
\epsilon:=\|(\mathbf{1}-|\mathrm{GS}\rangle\langle\mathrm{GS}|)\left|\widetilde{\mathrm{GS}}_{A}\right\rangle \|,
$$

where $\left|\widetilde{\mathrm{GS}}_{A}\right\rangle$ is the state adiabatically prepared in finite time $T$ and continuously, rather than in infinite time as in (10) or discretely as in (14). If the mixing between the ground state and the first excited state is the dominant source of the adiabatic error $\epsilon$, then it can be bounded as

$$
\epsilon \lesssim \max _{t} \frac{\left\|d H_{A} / d t\right\|}{\Delta^{2}}
$$

\footnotetext{
${ }^{13} \mathrm{~A}$ more detailed analysis shows that the (nonmonotonic) dependence of the systematic error on $g \ell$ cannot be understood purely in terms of the adiabatic error, and that we need to take into account the Trotter error.
} 
where $\Delta$ denotes the energy gap between the ground state and the first excited state. We note that $d H_{A} / d t \propto 1 / T$ when $H_{A}$ depends on $t$ only through $t / T$. Generically, we expect that larger values of $\left(w, J, m, q, \theta_{0}, \ell\right)$ lead to a larger value of $\left\|d H_{A} / d t\right\|$, and that a larger value of $L$ and a smaller value of $a$ lead to a smaller value of $\Delta$.

The density plots in Fig. 4 show the energy difference between the ground state and the first excited state. The energies are taken from the spectrum of the adiabatic Hamiltonian $H_{A}(t)$, which depends on $t$ through the following $t$-dependent parameters $(0 \leq t \leq T)$ : fermion mass $m_{0}(1-t / T)+m t / T$, inverse lattice spacing $w t / T$, and probe charge $q t / T$. Each horizontal path with increasing $t$ corresponds to an adiabatic variation of the parameters (13) with the probe charge $q$ of the target Hamiltonian fixed. For the adiabatic process along the horizontal path passing the region with small excitation energy (blue), a longer adiabatic time is likely to be required in order to prepare the corresponding target state with a given precision. In general, however, higher excited states, whose effects cannot be read off from Fig. 4, also contribute to the error in the adiabatic approximation and correct the inequality (A5). The region with small excitation energy appears for a larger value of $q$ and the region tends to spread as $\ell / a$ increases. This suggests that larger $\ell$ requires longer adiabatic time $T$.

Indeed in the left panel of Fig. 5, we find (for a fixed mass $m / g=0.25)$ that a larger value of $\ell / a(\gtrsim 14)$ requires a longer adiabatic time to achieve desired precision. The quantum simulation results obtained by adiabatic preparation approach the exact diagonalization results for large $T$, linearly in $1 / T$. This is likely due to a small energy-gap we expect for moderately large $g \ell$.

In the right panel of Fig. 5, we depict the energy density as a function of $\theta_{0}$. For $\delta t=0.3 g^{-1}$ and $0.2 g^{-1}$ the quantum simulation results are clearly not invariant under $\theta_{0} \rightarrow-\theta_{0}$, while the data obtained for $\delta t=0.1 g^{-1}$ are almost invariant and reproduce the exact diagonalization results. Exact diagonalization results shown there are for states within the same $\mathrm{U}(1)$ charge $\left(Q=\sum_{n} Z_{n}=-1\right)$ sector as the
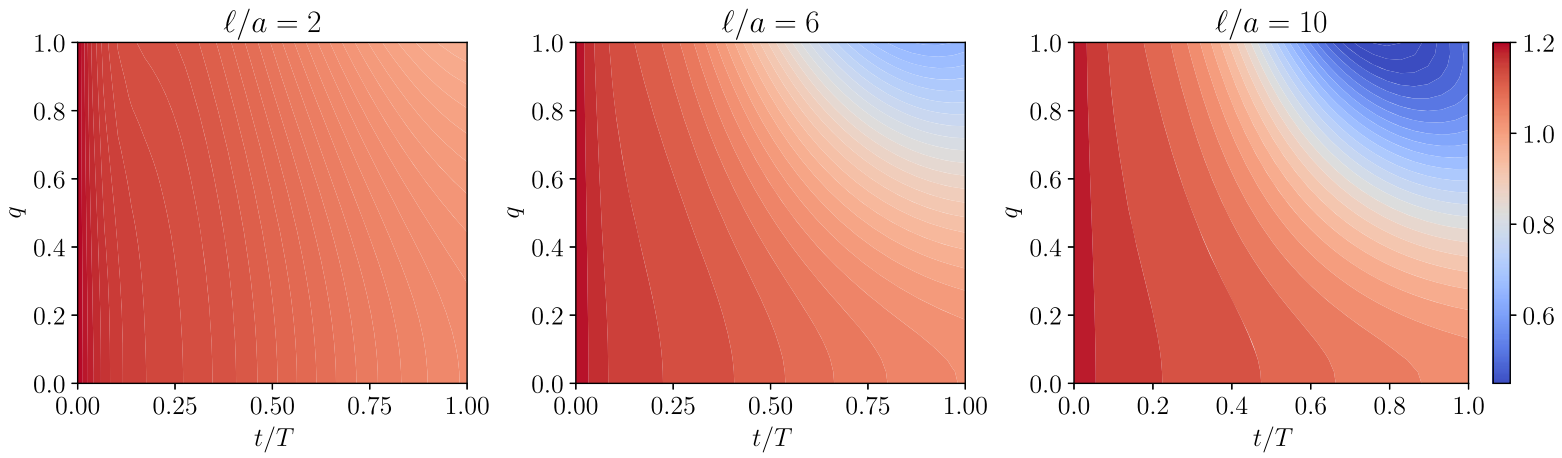

FIG. 4. Density plots of the eigenenergy of $H_{A}(t) / g$ for the first excited state relative to the ground state (both states in the $Q=-1$ sector) computed for $N=15, m_{0} / g=0.5, m / g=0.15, a g=0.4, \theta_{0}=0$, and $q=1$. Each panel shows the result for the indicated value of $\ell$.
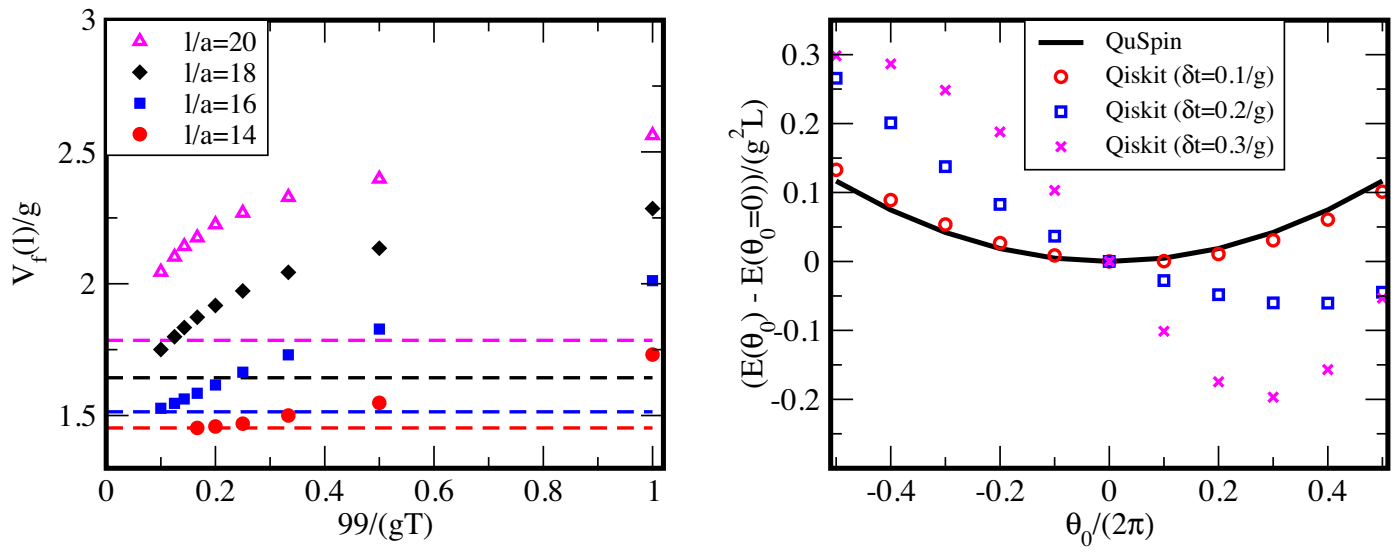

FIG. 5. Left: dependence of the quantum simulation result for $V_{f}(\ell)$ on the adiabatic time $T$ for $N=21, m / g=0.25, q=1, \theta_{0}=0$, and for the fixed value $\delta t=0.3 g^{-1}$. The dashed lines represent the results calculated by exact diagonalization. Right: dependence of the quantum simulation result for $E\left(\theta_{0}\right) / g^{2} L=E\left(\theta_{0}, q=0, \ell=0\right) / g^{2} L$ on $\theta_{0}$ and $\delta t$ for $N=15, m / g=0.05, m_{0} / g=0.55, T=99 g^{-1}$ and for a small value $a=0.1 g^{-1}$. The exact diagonalization (QuSpin) result is represented by the solid curve. 
initial state $|1010 \ldots\rangle$ for adiabatic preparation. We confirmed that for $\theta_{0} / 2 \pi \leq-0.4$, there are states with a different value of $Q$ and with energies lower than the values shown in the figure, while for $-0.35 \leq \theta_{0} / 2 \pi \leq$ +0.5 there are no such states.

\section{APPENDIX B: QUANTUM CIRCUIT}

\section{Quantum operations for state preparation}

All the quantum operations used in the main text consist of the following three elementary gates.

(i) Hadamard gate.

$$
H=\frac{1}{\sqrt{2}}\left(\begin{array}{cc}
1 & 1 \\
1 & -1
\end{array}\right)
$$

(ii) Phase (Z-rotation) gate.

$$
R_{Z}(\theta)=\mathrm{e}^{-\mathrm{i} \frac{\theta}{2} Z}=\left(\begin{array}{cc}
\mathrm{e}^{-\mathrm{i} \frac{\theta}{2}} & 0 \\
0 & \mathrm{e}^{\mathrm{i} \frac{\theta}{2}}
\end{array}\right)
$$

(iii) CNOT $(C X)$ gate.

$$
\underset{\square}{\square}=\left(\begin{array}{llll}
1 & 0 & 0 & 0 \\
0 & 1 & 0 & 0 \\
0 & 0 & 0 & 1 \\
0 & 0 & 1 & 0
\end{array}\right)
$$

Given a 2-qubit state on lattice sites labeled by $n \in$ $\{0,1\}$ we decompose $\mathrm{e}^{-\mathrm{i} \alpha\left(X_{0} X_{1}+Y_{0} Y_{1}\right)}$ and $\mathrm{e}^{-\mathrm{i} \alpha Z_{0} Z_{1}}$ in terms of the elementary gates defined above.

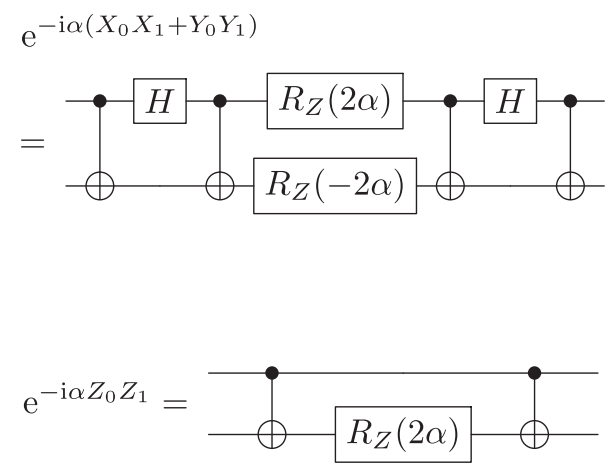

Here the top and bottom lines correspond to $n=0$ and $n=1$ respectively, and $\alpha$ is a real parameter.

The initial state $\left|\mathrm{GS}_{0}\right\rangle=|1010 \ldots\rangle$ in the adiabatic time evolution can be simply constructed as

$$
\left|\mathrm{GS}_{0}\right\rangle=\prod_{j=0}^{\left\lfloor\frac{N-1}{2}\right\rfloor} X_{2 j}|00 \ldots\rangle
$$

Here $\lfloor x\rfloor$ denotes the largest integer smaller than or equal to $x$.

\section{Measurements of $H_{X Y}^{(0)}, H_{X Y}^{(1)}, H_{Z}$}

We spell out the measurement protocol used to compute the Hamiltonian expectation value and its statistical uncertainty for a quantum state of interest. We consider the case where the state is a 4-qubit state for the sake of concreteness. The corresponding lattice sites are labeled by $n \in\{0,1,2,3\}$.

The term $H_{X Y}^{(0)}$ consists of the operators

$$
\left\{X_{0} X_{1}, Y_{0} Y_{1}, X_{2} X_{3}, Y_{2} Y_{3}\right\} .
$$

Noting that $^{14}$

$$
\begin{aligned}
& H_{i} C X_{i j}\left(X_{i} X_{j}\right) C X_{i j} H_{i}=H_{i}\left(X_{i} I_{j}\right) H_{i}=Z_{i} I_{j}, \\
& H_{i} C X_{i j}\left(Y_{i} Y_{j}\right) C X_{i j} H_{i}=-H_{i}\left(X_{i} Z_{j}\right) H_{i}=-Z_{i} Z_{j},
\end{aligned}
$$

the expectation values that we wish to measure can be expressed as

$$
\begin{aligned}
& \left\langle X_{i} X_{j}\right\rangle=\left\langle C X_{i j} H_{i}\left(Z_{i} I_{j}\right) H_{i} C X_{i j}\right\rangle, \\
& \left\langle Y_{i} Y_{j}\right\rangle=-\left\langle C X_{i j} H_{i}\left(Z_{i} Z_{j}\right) H_{i} C X_{i j}\right\rangle,
\end{aligned}
$$

where $I_{i}$ stands for the identity operator acting on $i$ th site. These quantities can be read off by the following circuit.

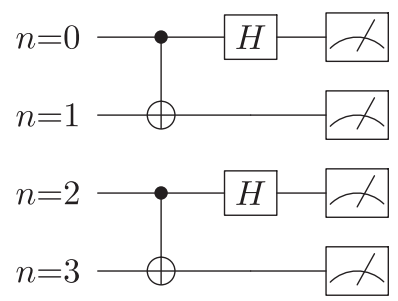

The four operations at the right end are the classical measurements in the $Z$ basis. Having obtained the counts of the bit strings " $b_{0} b_{1} b_{2} b_{3}$ " with $b_{i} \in\{0,1\}$ from the measurements, we calculate the expectation values from these samples as

\footnotetext{
${ }^{14}$ We employ the following identities repeatedly:

$$
\begin{aligned}
& C X_{i j}\left(X_{i} Y_{j}\right) C X_{i j}=Y_{i} Z_{j}, C X_{i j}\left(Y_{i} X_{j}\right) C X_{i j}=Y_{i} I_{j}, \\
& C X_{i j}\left(X_{i} X_{j}\right) C X_{i j}=X_{i} I_{j}, C X_{i j}\left(Y_{i} Y_{j}\right) C X_{i j}=-X_{i} Z_{j} .
\end{aligned}
$$
}




$$
\begin{aligned}
\left\langle X_{0} X_{1}\right\rangle_{b} & =\sum_{b_{n}}\left(1-2 b_{0}\right) \frac{\text { counts }_{b_{0} b_{1} b_{2} b_{3}}}{n_{\text {shots }}}, \\
\left\langle Y_{0} Y_{1}\right\rangle_{b} & =-\sum_{b_{n}}\left(1-2 b_{0}\right)\left(1-2 b_{1}\right) \frac{\text { counts }_{b_{0} b_{1} b_{2} b_{3}}}{n_{\text {shots }}}, \\
\left\langle X_{2} X_{3}\right\rangle_{b} & =\sum_{b_{n}}\left(1-2 b_{2}\right) \frac{\text { counts }_{b_{0} b_{1} b_{2} b_{3}}}{n_{\text {shots }}}, \\
\left\langle Y_{2} Y_{3}\right\rangle_{b} & =-\sum_{b_{n}}\left(1-2 b_{2}\right)\left(1-2 b_{3}\right) \frac{\text { counts }_{b_{0} b_{1} b_{2} b_{3}}}{n_{\text {shots }}} .
\end{aligned}
$$

Here $n_{\text {shots }}$ denotes the number of times the circuit is executed, ${ }^{15}$ and counts ${ }_{b_{0} b_{1} b_{2} b_{3}}$ denotes the number of times the bit string " $b_{0} b_{1} b_{2} b_{3}$ " is observed. The brackets $\langle\bullet\rangle_{b}$ stand for the expectation values from samples measured by the circuits (B9).

The term $H_{X Y}^{(1)}$ consists of the operators

$$
\left\{X_{1} X_{2}, Y_{1} Y_{2}\right\}
$$

Hence, the following measurement will do for the computation of their expectation values.

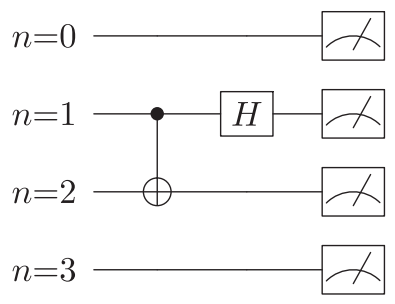

Given the counts of the bit strings " $b_{0} b_{1} b_{2} b_{3}$ ", we evaluate expectation values from samples as

$$
\begin{aligned}
\left\langle X_{1} X_{2}\right\rangle_{\sharp} & =\sum_{b_{n}}\left(1-2 b_{1}\right) \frac{\text { counts }_{b_{0} b_{1} b_{2} b_{3}}}{n_{\text {shots }}}, \\
\left\langle Y_{1} Y_{2}\right\rangle_{\sharp} & =-\sum_{b_{n}}\left(1-2 b_{1}\right)\left(1-2 b_{2}\right) \frac{\text { counts }_{b_{0} b_{1} b_{2} b_{3}}}{n_{\text {shots }}} .
\end{aligned}
$$

Here the brakets $\langle\bullet\rangle_{\sharp}$ stand for the expectation values from samples measured by the circuits (B11).

For $H_{Z}$, the measurements in the computational basis allow us to compute

$$
\begin{aligned}
\left\langle Z_{0}\right\rangle_{\natural} & =\sum_{b_{n}}\left(1-2 b_{0}\right) \frac{\text { counts }_{b_{0} b_{1} b_{2} b_{3}}}{n_{\text {shots }}}, \\
\left\langle Z_{0} Z_{1}\right\rangle_{\natural} & =\sum_{b_{n}}\left(1-2 b_{0}\right)\left(1-2 b_{1}\right) \frac{\text { counts }_{b_{0} b_{1} b_{2} b_{3}}}{n_{\text {shots }}},
\end{aligned}
$$

where brackets $\left\langle\bullet_{\natural}\right.$ denote the expectation values from samples measured in the computational basis. Combining

\footnotetext{
${ }^{15}$ Shots of microwave pulses execute quantum circuits on a real superconducting quantum computer.
}

these results leads to the expectation value of the total Hamiltonian (11).

We can also evaluate statistical uncertainties from the data obtained for computing the vacuum expectation value of the Hamiltonian as follows. First, we compute the expectation values of the squares of each term in the Hamiltonian from those data. For example, the term $\left(H_{X Y}^{(0)}\right)^{2}$ contains the term $X_{0} X_{1} X_{2} X_{3}$ and its expectation value is given by

$$
\left\langle X_{0} X_{1} X_{2} X_{3}\right\rangle_{b}=\sum_{b_{n}}\left(1-2 b_{0}\right)\left(1-2 b_{2}\right) \frac{\text { counts }_{b_{0} b_{1} b_{2} b_{3}}}{n_{\text {shots }}}
$$

Other terms in $\left\langle\left(H_{X Y}^{(0)}\right)^{2}\right\rangle_{b},\left\langle\left(H_{X Y}^{(1)}\right)^{2}\right\rangle_{\sharp},\left\langle\left(H_{Z}^{(0)}\right)^{2}\right\rangle_{\natural}$ can be evaluated in a similar manner. We then obtain the statistical uncertainty $\delta_{\text {stat }} E$ from these quantities as

$$
\left(\delta_{\text {stat }} E\right)^{2}=\frac{(\hat{\delta} E)^{2}}{n_{\text {shots }}-1}
$$

where $^{16}$

$$
\begin{aligned}
(\hat{\delta} E)^{2}:= & \left\langle\left(H_{X Y}^{(0)}\right)^{2}\right\rangle_{b}+\left\langle\left(H_{X Y}^{(1)}\right)^{2}\right\rangle_{\sharp}+\left\langle\left(H_{Z}^{(0)}\right)^{2}\right\rangle_{\natural} \\
& -\left(\left\langle H_{X Y}^{(0)}\right\rangle_{b}\right)^{2}-\left(\left\langle H_{X Y}^{(1)}\right\rangle_{\sharp}\right)^{2}-\left(\left\langle H_{Z}\right\rangle_{\natural}\right)^{2} .
\end{aligned}
$$

\section{Resource estimation}

Let us estimate the computational resource required for our simulation, closely following [87]. The circuit (B4) has four CNOT gates and two $R_{Z}$-gates, while the circuit (B5) has two CNOT gates and one $R_{Z}$-gate. Each step in the Suzuki-Trotter decomposition (14) has $2(N-1)$ subcircuits of the form (B4) and $\frac{1}{2}(N-1)(N-2)$

$$
\begin{aligned}
& { }^{16} \text { Let us consider more general cases first. Suppose that we } \\
& \text { want to evaluate the sum of noncommutative operators } A, B \text {, and } \\
& \text { we measure two operators independently. Then, the variance of } \\
& A+B \text { is evaluated as } \\
& \qquad \sum_{A, B} f(A) g(B)(A+B)^{2}-\left(\sum_{A} f(A)\right)^{2} \\
& \quad-2\left(\sum_{A} f(A)\right)\left(\sum_{B} g(B)\right)-\left(\sum_{B} g(B)\right)^{2} \\
& =\sum_{A} f(A) A^{2}+\sum_{B} g(B) B^{2} \\
& -\left(\sum_{A} f(A) A\right)^{2}-\left(\sum_{B} g(B) B\right)^{2},
\end{aligned}
$$

where $f(A), g(B)$ are the distribution functions for $A$ and $B$. So we do not need to consider cross terms. In our protocol, we independently measure the expectation values of $H_{X Y}^{(0)}, H_{X Y}^{(1)}$ and $H_{Z}^{(0)}$, thus the statistical uncertainty of the total energy can be evaluated as (B12) and (B13) without cross terms. 
subcircuits of the form (B5), and $N R_{Z}$-gates besides. Thus, for odd $N$, we need $(N-1)(N+6)$ CNOT gates and $\frac{1}{2}\left(N^{2}+7 N-6\right) R_{Z}$-gates for each step.

As mentioned in Sec. V, CNOT gates are the main source of errors in NISQ devices. Thus the number $(N-1)(N+$ 6) of CNOT gates is crucial for implementation on NISQ devices. On the other hand, in most approaches to faulttolerance $[88,89]$, the most costly components are nonClifford operations such as $T$-gates, where $T=\operatorname{diag}\left(1, \mathrm{e}^{\mathrm{i} \pi}\right)$ in a matrix representation. In our algorithm $T$-gates appear only in implementing $R_{Z}$-gates. They can be implemented by using the repeat-until-success method $[90,91]$ with $1.15 \log _{2}(2 / \delta) T$-gates within an accuracy $\delta$ :

$$
\left\|R_{Z}-\tilde{R}_{Z}\right\|<\delta
$$

where $\tilde{R}_{Z}$ approximates $R_{Z}$-gates in a fault-tolerant manner and $\|\cdot\|$ stands for the spectral norm. Thus we need $0.575\left(N^{2}+7 N-6\right) \log _{2}\left(\left(N^{2}+7 N-6\right) / \delta\right) \quad T$-gates to implement each step in the Suzuki-Trotter decomposition (14).

In our simulations, the computation of the potential $V_{f}$ within the $\mathcal{O}(10 \%)$ accuracy requires $n_{\text {shots }}=4 \times 10^{5}$ measurements for a noninteger probe charge. In our quantum simulation on a classical simulator, it takes about 3 hours for $N=21$ to obtain the potential in the range $0 \leq \ell / a \leq 20$ (11 data points) with $n_{\text {shots }}=10^{5}$ on a typical personal computer (such as iMac).

\section{APPENDIX C: COMMENTS ON SYMMETRIES}

We comment on discrete symmetries in the lattice theory. In the continuum theory, the net effect of the parity (or charge conjugation) transformation is to flip the sign of $\theta_{0}$ because it acts as $F_{01} \rightarrow-F_{01}$. Therefore, the continuum theory in the absence of probes is manifestly parity invariant at $\theta_{0}=0$. On a space without boundary, the theory at $\theta_{0}=\pi$ also has parity invariance because of the periodicity $\theta_{0} \sim \theta_{0}+2 \pi$ while in the case with boundaries, the theories at $\theta_{0}=\pi$ and $-\pi$ differ by boundary terms in the action.

The situation in the lattice theory is more intricate. An analog of the parity transformation on the lattice is

$$
\begin{aligned}
& \chi_{n} \rightarrow i(-1)^{n} \chi_{N-1-n}, \quad U_{n} \rightarrow U_{N-2-n}^{-1}, \\
& L_{n} \rightarrow-L_{N-2-n} .
\end{aligned}
$$

For the periodic boundary condition, in which $N$ is always even, the periodic analog of the lattice Hamiltonian (4) is invariant under this transformation for $\theta_{0}=0$ and $\pi .{ }^{17}$ With the open boundary condition, the situation is different for odd $N$ and even $N$. For odd $N$, the net effect of the

\footnotetext{
${ }^{17}$ The $(-1)^{n} / 2$ term in the Gauss law constraint (5) violates the invariance under (C1) for even $N$.
}

transformation $(\mathrm{C} 1)$ is to flip the sign of the theta angle as in the case of the continuum theory: it is parity invariant for $\theta_{0}=0$, and the cases with $\theta_{0}=\pi$ and $-\pi$ differ only by boundary terms. However, for even $N$, the transformation (C1) flips not only the theta angle but also the mass $m$. This implies that the lattice theory with even $N$ and nonzero mass does not have parity symmetry for any value of $\theta_{0}$. Thus we take $N$ to be odd in this work. In the presence of symmetrically located probe charges in (3), the net effect of the parity transformation $(\mathrm{C} 1)$ is to flip the signs of both $\theta_{0}$ and $q$.

With the choice of the decomposition (11), the time evolution in (14) respects the $U(1)$ symmetry generated by $Q=\sum_{n=0}^{N-1} Z_{n},{ }^{18}$ while it violates the parity symmetry, which is only approximately restored for small $\delta t$. Whether it is $\mathrm{U}(1)$ or parity, a symmetry can be a useful property for simulation (see, e.g., [92]). In general an adiabatic process prepares a state within the same charge sector as the initial state. We checked by exact diagonalization that the lowestenergy states among all charge sectors do have $Q=-1$ for the parameters discussed in Figs. 1 and 2, and in Tables I and II in Appendix A. But for some of the parameters considered in Figs. 3 and 5, we found that the lowestenergy states have different values of $Q$.

\section{APPENDIX D: CONTINUUM SCHWINGER MODEL}

\section{Summary of results}

We summarize some analytic results for two probe charges $\pm q$ separated by distance $\ell$ in the continuum Schwinger model. Some of the results are new.

We begin with the theory in the infinite-volume limit. In this case, there are some results by mass perturbation theory in the literature $[9,81,93]$. The potential in the massless $(m=0)$ theory is given by [81]

$$
V^{(0)}(\ell)=\frac{q^{2} g^{2}}{2 \mu}\left(1-\mathrm{e}^{-\mu \ell}\right),
$$

where $\mu=g / \sqrt{\pi}$. The $\mathcal{O}(m)$ correction to the potential for $\ell \gg 1 / \mu$ is given by

$V^{(1)}(\ell)=m \Sigma(1-\cos (2 \pi q)) \ell+c_{q} m+o(1 / \ell)$

where $\Sigma=e^{\gamma} g /\left(2 \pi^{3 / 2}\right)$. The first term giving the string tension has been known in the literature (see e.g., [9]) while the second term has not. In Appendix D 2, we find that the constant $c_{q}$ is given by

\footnotetext{
${ }^{18}$ See [17] for another decomposition that preserves U(1).
} 


$$
\begin{aligned}
c_{q}= & \frac{2 \mathrm{e}^{\gamma}}{\pi} \cos \left(\theta_{0}+\pi q\right)[\cos (\pi q) \operatorname{Cin}(\pi q) \\
& -\sin (\pi q) \operatorname{Si}(\pi q)],
\end{aligned}
$$

where $\operatorname{Cin}(z):=\int_{0}^{z} d s[1-\cos (s)] / s \quad$ and $\quad \operatorname{Si}(z):=$ $\int_{0}^{z} d s \sin (s) / s$ are known as the cosine and sine integral functions, respectively. ${ }^{19}$ Note that $c_{q}$ is nonzero even when $q$ is an integer. This implies that the potential for large $\ell$ in integer $q$ case approaches a nonzero constant which is given by $c_{q} m$ up to an $\mathcal{O}\left(m^{2}\right)$ correction. Therefore the value of the screening potential on the plateau in the massive case differs from the one in the massless case, as can be confirmed for the analytic curves on the right panel of Fig. $1 .^{20}$ The $\mathcal{O}\left(\mathrm{m}^{2}\right)$ correction for $\ell \gg 1 / \mu$ is [93]

$V^{(2)}(\ell)=m^{2}\left[\pi\left(\frac{\Sigma}{2 g^{2}}\right)^{2} \mu_{0}^{2} \mathcal{E}_{+}(1-\cos (4 \pi q)) g \ell+\mathcal{O}(1)\right]$,

where $\mu_{0}^{2} \mathcal{E}_{+} \simeq-8.9139 .^{21}$ This formula is consistent with the expectation that the string tension vanishes exactly when $q$ is an integer [8].

Next let us consider the Schwinger model on the finite interval $[0, L]$. As explained in Appendix D 2, we take an appropriate boundary condition, which corresponds to the continuum limit of the lattice model studied in the main text. For this boundary condition, we derive the following results for the probe charges $\pm q$ placed at $x=(L \mp \ell) / 2$. For $m=0$, the ground state energy is

$$
E_{f}^{(0)}\left(\theta_{0}, q, \ell\right):=\sum_{n=1}^{\infty} \frac{L \mu^{2}}{16 \pi} \frac{k_{n}^{2}}{\omega_{n}^{2}}\left(\Theta_{n}^{(q)}\right)^{2}
$$

where

$$
\begin{aligned}
\Theta_{n}^{(q)}= & \frac{1-(-1)^{n}}{n}\left(\frac{2 \theta_{0}}{\pi}+(-1)^{\frac{n-1}{2}} 4 q \sin \left(\frac{\pi n \ell}{2 L}\right)\right) \\
& +\frac{1+(-1)^{n}}{n} .
\end{aligned}
$$

For $\theta_{0}=0$, the potential $V_{f}^{(0)}(\ell):=E_{f}^{(0)}(0, q, \ell)-$ $E_{f}^{(0)}(0, q, 0)$ simplifies to

\footnotetext{
${ }^{19}$ The function $\operatorname{Cin}(z)$ is related to a more common function $\operatorname{Ci}(z):=-\int_{z}^{\infty} d s \cos (s) / s$ as $\operatorname{Cin}(z)=\gamma+\log z-\operatorname{Ci}(z)$.

${ }^{20}$ For $m / g=0.2, c_{q=1} m / g \simeq 0.37$, which is the difference between the limiting values (for large $g \ell$ ) of the dashed curves in green and purple in Fig. 1.

${ }^{21}$ The precise definition is given by Eq. (64) in [93] as $\mu_{0}^{2} \mathcal{E}_{+}=$ $2 \pi \int_{0}^{\infty} \operatorname{drr}\left(\mathrm{e}^{-2 K_{0}(r)}-1\right)$ with the modified Bessel function $K_{0}(r)$ of the second kind.
}

$$
V_{f}^{(0)}(\ell)=\frac{q^{2} g^{2}}{2 \mu} \frac{\left(1-\mathrm{e}^{-\mu \ell}\right)\left(1+\mathrm{e}^{-\mu(L-\ell)}\right)}{1+\mathrm{e}^{-\mu L}} .
$$

One can easily see that this reproduces (D1) in the infinitevolume limit $L \rightarrow \infty$ with $1 \ll \mu \ell \ll \mu L$. The $\mathcal{O}(m)$ correction to the energy is given by

$$
\begin{aligned}
E_{f}^{(1)}\left(\theta_{0}, q, \ell\right)= & -m \Sigma \int_{0}^{L} d x \lambda(x) \\
& \times \cos \left[2 \sqrt{\pi} \phi_{0}-\sum_{n=1}^{\infty} \frac{\mu^{2} \Theta_{n}^{(q)}}{\mu^{2}+k_{n}^{2}} \sin \left(k_{n} x\right)\right],
\end{aligned}
$$

where $k_{n}=\pi n / L$,

$\lambda(x):=\lim _{\Lambda \rightarrow \infty} \exp \left[\sinh ^{-1}\left(\frac{\Lambda}{\mu}\right)-\sum_{n=1}^{\lfloor L \Lambda / \pi\rfloor} \frac{2 \pi}{L} \frac{\sin ^{2}\left(k_{n} x\right)}{\sqrt{\mu^{2}+k_{n}^{2}}}\right]$,

$\Lambda$ is a UV cutoff sent to infinity, and $\lfloor x\rfloor$ denotes the largest integer smaller than or equal to $x$. The $\mathcal{O}(m)$ correction to the potential for $\theta_{0}=0$ is by definition

$$
V_{f}^{(1)}(\ell):=E_{f}^{(1)}(0, q, \ell)-E_{f}^{(1)}(0, q, 0) .
$$

We also show in Appendix D 2 that for $m / g \gg 1$ and for $\theta_{0}=0$, the potential $V_{f}(\ell)$ becomes

$$
\frac{q^{2} g^{2}}{2}\left[1-\frac{\mathrm{e}^{-\gamma} g}{2 \sqrt{\pi} m}+\mathcal{O}\left((g / m)^{2}\right)\right] \ell .
$$

The leading term is the Coulomb potential of the pure U(1) gauge theory as expected.

\section{Bosonized Schwinger model on an interval}

Here we study the bosonized Schwinger model on an interval and derive some of the analytic results used in the main text and summarized Appendix D 1. Let us consider the Schwinger model on the spacetime $\mathbb{R} \times[0, L]$. We parametrize the time by $t=x^{0}$ and the interval $[0, L]$ by $x=x^{1}$. After bosonization the theory with a positiondependent theta angle $\Theta(x)$ is described by the Lagrangian density

$$
\begin{aligned}
\mathcal{L}_{b}= & -\frac{1}{4} F_{\mu \nu} F^{\mu \nu}+\frac{g}{4 \pi} \Theta(x) \epsilon^{\mu \nu} F_{\mu \nu}+\frac{g}{\sqrt{\pi}} \epsilon^{\mu \nu} A_{\mu} \partial_{\nu} \phi \\
& +\frac{1}{2} \partial_{\mu} \phi \partial^{\mu} \phi+m g \frac{e^{\gamma}}{2 \pi^{3 / 2}} \cos (2 \sqrt{\pi} \phi) .
\end{aligned}
$$

We first specify the boundary conditions for the fields. For $\phi$, we impose

$$
\left.\phi\right|_{x=0}=\sqrt{\pi} w_{0},\left.\quad \phi\right|_{x=L}=\sqrt{\pi} w_{1},
$$


where $w_{0}$ and $w_{1}$ are real (not necessarily integral) constants specifying the boundary conditions. In the main text we set these constants to the specific values $w_{0}=1 / 4$ and $w_{1}=-1 / 4$ for the reasons we explain below (D23). Regarding the gauge field, it must be consistent with the Gauss law constraint $\delta\left(\int d^{2} x \mathcal{L}_{b}\right) / \delta A_{0}=0$, which reads

$$
\partial_{1}\left(F_{01}+\frac{g}{2 \pi}(\Theta+2 \sqrt{\pi} \phi)\right)=0 .
$$

This is solved by

$$
F_{01}=\frac{g}{2 \pi}(\Theta+2 \sqrt{\pi} \phi)+f\left(x^{0}\right),
$$

where $f\left(x^{0}\right)$ is $x$-independent. As in Section II we work in the temporal gauge $A_{0}=0$. We choose the boundary condition on $A_{1}$ such that $f\left(x^{0}\right)=0$.

Denoting the canonical momentum conjugate to $\phi$ by $\Pi_{\phi}$, we get the Hamiltonian density

$$
\begin{aligned}
\mathcal{H}_{b}(x)= & \frac{1}{2} \Pi_{\phi}^{2}+\frac{1}{2}\left(\partial_{x} \phi\right)^{2}+\frac{g^{2}}{2 \pi}\left(\phi+\frac{\Theta(x)}{2 \sqrt{\pi}}\right)^{2} \\
& -m g \frac{\mathrm{e}^{\gamma}}{2 \pi^{3 / 2}} \cos (2 \sqrt{\pi} \phi)
\end{aligned}
$$

and the Hamiltonian $H_{b}=\int_{0}^{L} d x \mathcal{H}_{b}(x)$. We note that the numerical coefficient $\mathrm{e}^{\gamma} / 2 \pi^{3 / 2}$ for the cosine terms in (D12) and (D16) is appropriate only if " $\cos (2 \sqrt{\pi} \phi)$ " in (D16) is interpreted as normal-ordered with mass $\mu=g / \sqrt{\pi}$ on an infinite spatial line. This will be important below.

We now set $m=0$ to compute the energy by the mass perturbation theory. Let us define $\phi_{0}(x)$ and $\hat{\phi}(x)$ by

$$
\begin{aligned}
\phi_{0}(x) & =\sqrt{\pi} w_{0}+\sqrt{\pi}\left(w_{1}-w_{0}\right) \frac{x}{L}, \\
\hat{\phi}(x) & =\phi(x)-\phi_{0}(x) .
\end{aligned}
$$

We also define

$$
\hat{\Theta}(x) \equiv \Theta(x)+2 \sqrt{\pi} \phi_{0}(x) .
$$

We expand the operators in terms of the Fourier coefficients $\phi_{n}, \Pi_{n}, \Theta_{n}$ as

$$
\begin{array}{ll}
\hat{\phi}(x)=\sum_{n=1}^{\infty} \phi_{n} \sin \left(k_{n} x\right), \quad \Pi_{\phi}(x)=\sum_{n=1}^{\infty} \Pi_{n} \sin \left(k_{n} x\right), \\
\hat{\Theta}(x)=\sum_{n=1}^{\infty} \Theta_{n} \sin \left(k_{n} x\right) .
\end{array}
$$

Here we extended $\hat{\Theta}(x)$ to a (possibly discontinuous) odd periodic function with period $2 L$. We have the canonical commutation relations $\left[\phi_{n}, \Pi_{n^{\prime}}\right]=(2 \mathrm{i} / L) \delta_{n n^{\prime}}$. The Hamiltonian can be written as

$$
H_{b}=\frac{\pi\left(w_{1}-w_{0}\right)^{2}}{2 L}+\sum_{n=1}^{\infty}\left[\omega_{n}\left(a_{n}^{\dagger} a_{n}+\frac{1}{2}\right)+\frac{L \mu^{2}}{16} \frac{k_{n}^{2}}{\omega_{n}^{2}} \Theta_{n}^{2}\right],
$$

where $\omega_{n}=\sqrt{\mu^{2}+k_{n}^{2}}$ and

$$
a_{n}=\frac{\sqrt{L \omega_{n}}}{2}\left(\phi_{n}+\frac{\mu^{2} \Theta_{n}}{2 \sqrt{\pi} \omega_{n}^{2}}\right)+\frac{\mathrm{i}}{2} \sqrt{\frac{L}{\omega_{n}}} \Pi_{n}
$$

We have $\left[a_{n}, a_{n^{\prime}}^{\dagger}\right]=\delta_{n n^{\prime}}$. The ground state $|0\rangle$ satisfies $a_{n}|0\rangle=0$, and its energy is

$$
\left\langle 0\left|H_{b}\right| 0\right\rangle=\frac{\pi\left(w_{1}-w_{0}\right)^{2}}{2 L}+\sum_{n=1}^{\infty}\left(\frac{\omega_{n}}{2}+\frac{L \mu^{2}}{16 \pi} \frac{k_{n}^{2}}{\omega_{n}^{2}} \Theta_{n}^{2}\right) .
$$

The sum of the first term in the bracket is the ( $L$-dependent) Casimir energy. It is UV-divergent but independent of $\Theta$. Therefore we focus on the sum of the second term. We take

$$
\Theta(x)= \begin{cases}\theta_{0}+2 \pi q & \text { for } \frac{L-\ell}{2} \leq x \leq \frac{L+\ell}{2} \\ \theta_{0} & \text { otherwise. }\end{cases}
$$

The Fourier coefficients for $\hat{\Theta}$ are then

$$
\begin{aligned}
\Theta_{n}= & \frac{1-(-1)^{n}}{n}\left[\frac{2 \theta_{0}}{\pi}+(-1)^{\frac{n-1}{2}} 4 q \sin \left(\frac{\pi n \ell}{2 L}\right)\right] \\
& +4 \frac{w_{0}-(-1)^{n} w_{1}}{n}
\end{aligned}
$$

We claim that the lattice Schwinger model described in Sec. II corresponds, in the continuum limit, to the choice $w_{0}=1 / 4$ and $w_{1}=(1 / 2) Q+1 / 4$, where $Q:=\sum_{n=0}^{N-1} Z_{n}$. To see this, let us compare the Gauss law constraints $L_{n}-L_{n-1}=\left[Z_{n}+(-1)^{n}\right] / 2$ and (D14) in the spin and bosonized formulations, respectively. The correspondence $L_{n} \leftrightarrow(1 / g) F_{01}-(1 / 2 \pi) \Theta$ suggests that $\phi(x) / \sqrt{\pi}$ is the mean field (spatial average) for $(1 / 2) \sum_{i=0}^{n}\left[Z_{i}+(-1)^{i}\right]$. By the symmetry under $Z_{i} \rightarrow-Z_{i}$ present for $g=0$, the mean value of $Z_{i}$ on sites near (compared with $1 / g$ ) the boundary must vanish. The mean value of $(1 / 2) \sum_{i=0}^{n}(-1)^{i}$ is $1 / 4$. This leads to our claim, which can be explicitly confirmed by comparing the results for the charge density computed by DMRG and by bosonization [94].

We now restrict to odd $N$ and $Q=-1$, so that $w_{1}=-1 / 4$. Substituting the values of $w_{0}$ and $w_{1}$ to (D23) gives (D6). The $q$-dependent part of the ground state energy (D21) for $\theta_{0}=0$ 


$$
\frac{4 g^{2} q^{2}}{L} \sum_{j=0}^{\infty} \frac{\sin ^{2}\left(k_{2 j+1} \ell / 2\right)}{\omega_{2 j+1}^{2}}
$$

can be rewritten as the potential (D7) in the massless case. ${ }^{22}$

To derive the $\mathcal{O}(m)$ correction to the ground state energy, we now compute the chiral condensate in the massless theory. Upon bosonization it is given as

$$
\langle\bar{\psi} \psi(x)\rangle=-\frac{\mathrm{e}^{\gamma} g}{2 \pi^{3 / 2}}\left\langle 0\left|: \cos (2 \sqrt{\pi} \phi(x)):_{\infty}\right| 0\right\rangle,
$$

where the normal ordering $: \bullet:_{\infty}$ is taken with respect to the creation and annihilation operators on an infinite line, while $|0\rangle$ is the ground state on the interval. Let us denote the normal ordering with respect to $a_{n}$ in (D20) by : $\bullet:$ We find formally

$$
\begin{aligned}
& : \mathrm{e}^{ \pm 2 \mathrm{i} \sqrt{\pi} \phi(x)}:_{\infty}=\mathrm{e}^{ \pm 2 \mathrm{i} \sqrt{\pi} \phi(x)} \exp \left(\int_{0}^{\infty} \frac{d k}{\sqrt{\mu^{2}+k^{2}}}\right) \\
& : \mathrm{e}^{ \pm 2 \mathrm{i} \sqrt{\pi} \phi(x)}:=\mathrm{e}^{ \pm 2 \mathrm{i} \sqrt{\pi} \phi(x)} \exp \left[\sum_{n=1}^{\infty} \frac{2 \pi}{L} \frac{\sin ^{2}\left(k_{n} x\right)}{\sqrt{\mu^{2}+k_{n}^{2}}}\right]
\end{aligned}
$$

The right-hand sides of (D26) and (D27) involve UV divergent expressions. By introducing a cutoff, we obtain the well-defined relation

$$
: \mathrm{e}^{ \pm 2 \mathrm{i} \sqrt{\pi} \phi(x)}:_{\infty}=\lambda(x): \mathrm{e}^{ \pm 2 \mathrm{i} \sqrt{\pi} \phi(x)}:
$$

where $\lambda(x)$ is defined in (D9). Then (D25) gives ${ }^{23}$

$$
\langle\bar{\psi} \psi(x)\rangle=-\frac{\mathrm{e}^{\gamma} g}{2 \pi^{3 / 2}} \lambda(x) \cos \left[2 \sqrt{\pi} \phi_{0}-\sum_{n=1}^{\infty} \frac{\mu^{2} \Theta_{n}}{\omega_{n}^{2}} \sin \left(k_{n} x\right)\right] .
$$

With this, perturbation theory gives the correction (D8) to the ground state energy.

For a single probe defined by

$$
\Theta_{\text {probe }}(x):= \begin{cases}\theta_{0} & \text { for } x<0 \\ \theta_{0}+2 \pi q & \text { for } x>0\end{cases}
$$

we find, either from the general formula (D29) or by repeating the derivation,

\footnotetext{
${ }^{22}$ Let $\alpha$ be real and $b \in \mathbb{Z}$ satisfy $b \leq \alpha \leq b+1$. A useful formula $\sum_{j \in \mathbb{Z}} \frac{\mathrm{e}^{2 \pi i \alpha(j+1 / 2)}}{A^{2}+\pi^{2}(j+1 / 2)^{2}}=(-1)^{b} \frac{\sinh [(1+2 b-2 \alpha) A]}{A \cosh (A)}$ can be proved by the residue theorem and contour deformation.

${ }^{23}$ With the special values $w_{0}=1 / 4$ and $w_{1}=-1 / 4$, the chiral condensate $\langle\bar{\psi} \psi(x)\rangle$ is finite near the boundaries.
}

$$
\begin{aligned}
\langle\bar{\psi} \psi(x)\rangle_{\text {probe }}= & -\frac{\mathrm{e}^{\gamma} g}{2 \pi^{3 / 2}} \cos \left[\theta_{0}+\pi q\right. \\
& \left.+\operatorname{sgn}(x) \pi q\left(1-\mathrm{e}^{-\mu|x|}\right)\right] .
\end{aligned}
$$

Integrating, over $x \in(-\infty, 0]$ for example, the difference between (D31) and its asymptotic value gives

$$
\begin{array}{rl}
\int_{-\infty}^{0} & d x\left(\langle\bar{\psi} \psi(x)\rangle_{\text {probe }}+\frac{\mathrm{e}^{\gamma} g}{2 \pi^{3 / 2}} \cos \theta_{0}\right) \\
= & \frac{\mathrm{e}^{\gamma}}{2 \pi}\left[\cos \theta_{0} \operatorname{Cin}(\pi q)+\sin \theta_{0} \operatorname{Si}(\pi q)\right],
\end{array}
$$

where $\quad \operatorname{Cin}(z)=\int_{0}^{z} d s[1-\cos (s)] / s \quad$ and $\quad \operatorname{Si}(z)=$ $\int_{0}^{z} d s \sin (s) / s$. By considering two well-separated probes, it follows that the constant $c_{q}$ in (D2) is given by (D3).

Finally we consider the large mass limit $m / g \gg 1$ of (D16). We assume that $\left|w_{0}\right|,\left|w_{1}\right|<1 / 2$. The cosine term forces $\phi$ to be localized near $\phi=0$ in the bulk. Expanding the cosine in $\phi$, we can write the potential part of $\mathcal{H}_{b}$ as

$$
C_{1}\left(\phi+C_{2} \Theta\right)^{2}+\frac{g^{2}}{8 \pi^{2}}\left(1+\frac{\mathrm{e}^{-\gamma} g}{2 \sqrt{\pi} m}\right)^{-1} \Theta^{2}+\mathcal{O}\left(\phi^{3}\right),
$$

where $C_{1}=\mathcal{O}(\mathrm{m} / \mathrm{g})$ and $C_{2}=\mathcal{O}(\mathrm{g} / \mathrm{m})$ are constants. Substituting (D22) and repeating the analysis leading to (D21), we obtain the result (D11) for the potential $V_{f}(\ell)$.

\section{Volume dependence of the slope of the potential}

The results in Fig. 2 include finite-size corrections. We wish to estimate the number of qubits for which such corrections become negligible. Using our analytic formulas for $V_{f}^{(0)}+V_{f}^{(1)}$, we look for the values of volume $L$ where the slope (the first derivative) at an appropriate point accurately reproduces the string tension $\sigma^{(1)}$ in the infinite volume. For $m / g=0.2$ and $g a=0.4$, we find that with $L=13.6(N=35)$, the slope at $\ell=L / 2$ reproduces $\sigma^{(1)}$ within 5 percent, as can be seen from Fig. 7, where $(d / d \ell)\left(V_{f}^{(0)}+V_{f}^{(1)}\right) / g^{2}$ at $\ell=L / 2$ is plotted for several values of $N$. We thus expect that an ideal quantum simulation with $N=35$ or larger will exhibit reasonable agreement with the true (infinite-volume) string tension.

\section{APPENDIX E: MASS DEPENDENCE OF THE POTENTIAL}

Here we study the dependence of the potential $V_{f}(\ell)$ on the fermion mass $m$. We depict quantum simulation results and $\mathcal{O}(m)$ analytic predictions for $V_{f}(\ell)$ in Fig. $6 .{ }^{24}$ As in Sec. IV we take $a=0.4 g^{-1}, \delta t=0.3 g^{-1}, T=99 g^{-1}$ and $m_{0}=0.5 \mathrm{~g}$. We use the "snapshot" functionality of Qiskit to obtain quantum simulation results without statistical

\footnotetext{
${ }^{24}$ These simulation results include adiabatic errors. See Tables I and II for the systematic errors in similar settings.
} 

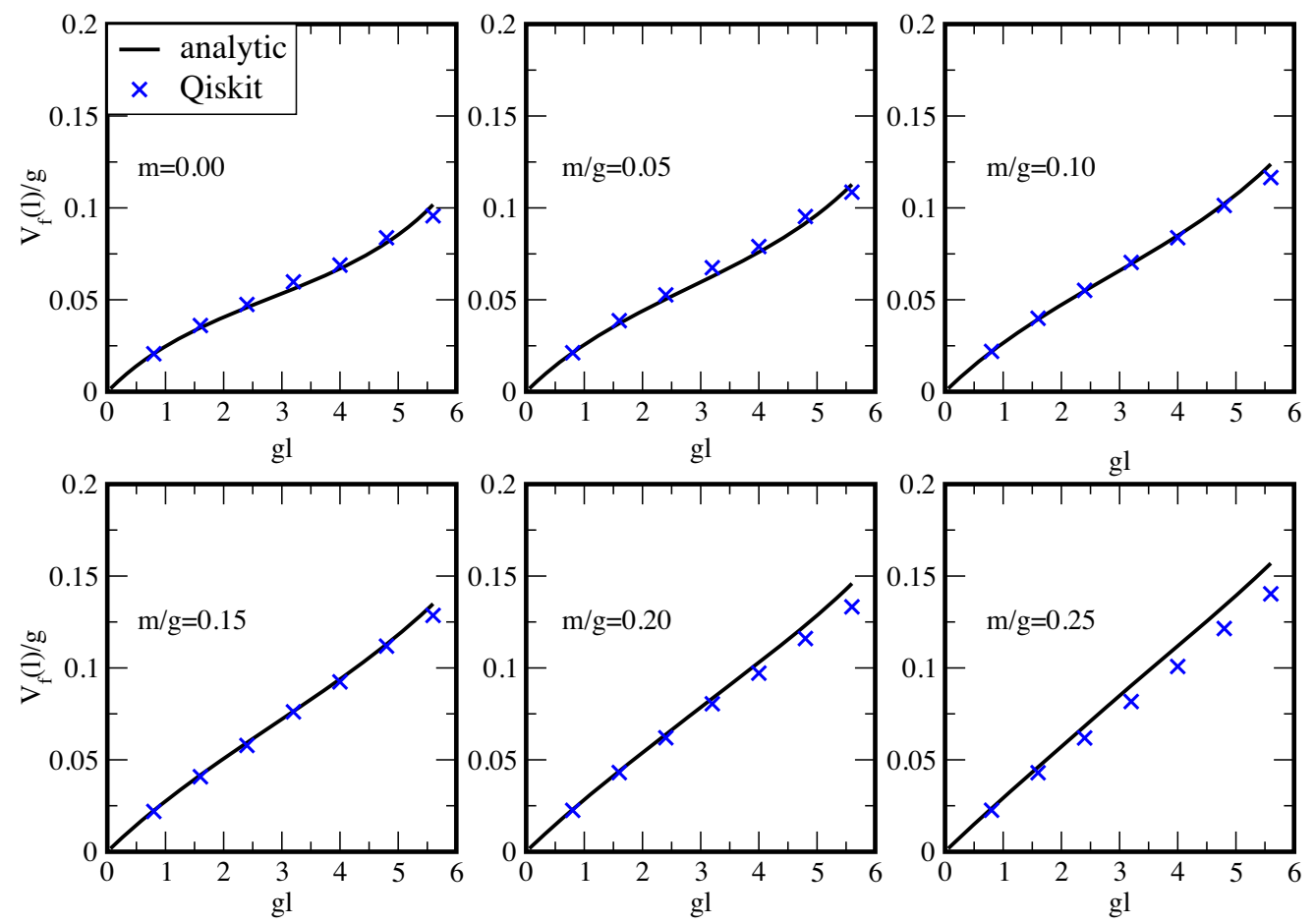

FIG. 6. Potential $V_{f}(\ell)$ for several values of $m / g$, and for $N=15, q=0.25, \theta_{0}=0$. The blue crosses represent the results of quantum simulation without statistical uncertainties. The black solid curves represent the $\mathcal{O}(m)$ analytic prediction $V_{f}^{(0)}(\ell)+V_{f}^{(1)}(\ell)$.

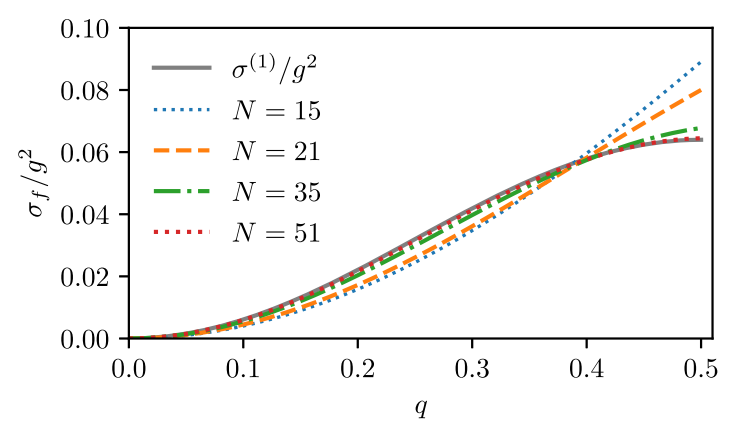

FIG. 7. The values of $(d / d \ell)\left(V_{f}^{(0)}+V_{f}^{(1)}\right) / g^{2}$ at $\ell=L / 2$ for $L=(N-1) a, m / g=0.2$, and $g a=0.4$, with the indicated values of $N$. We also plot $\sigma^{(1)} / g^{2}$ given in (17).

uncertainties. As we increase the mass within the range $m / g \lesssim 0.15$, the analytic results become less curved. At $m / g=0.2$ and 0.25 , the curves are almost straight lines.

In the small mass range $0<m / g \lesssim 0.15$, the simulation results match well the analytic predictions. For $0.2 \lesssim m / g \lesssim 0.25$, the differences between the simulation results and the $\mathcal{O}(\mathrm{m})$ analytic predictions are larger. If the systematic errors in our results are small, then this discrepancy should be due to finite $a$ effect or/and breaking of the approximation by the mass perturbation in this regime.

We expect that the latter is a dominant source of the discrepancy as follows. There is another numerical study [95] of the lattice Schwinger model by the density matrix renormalization group (DMRG) method, which takes the both continuum and infinite-volume limits. In [95], analogous deviations of the numerical results from the analytic $\mathcal{O}(m)$ results were found for similar values of $\mathrm{m} / \mathrm{g}$. Since the continuum limit is taken in [95], this implies that the mass perturbation theory in the regime $0.2 \lesssim m / g \lesssim 0.25$ is no longer reliable at least for large volume. Although it is nontrivial whether this is still true for the value of our volume, we regard this as indirect evidence of our expectation. Then our results in Fig. 6 likely give a prediction for the middle range of mass between small mass and massive limit. It would be important to check the consistency of the results between the quantum computing and the DMRG in such a nonperturbative mass regime in the future. 
[1] K. G. Wilson, Confinement of quarks, Phys. Rev. D 10, 2445 (1974).

[2] M. Creutz, Monte Carlo study of quantized SU(2) gauge theory, Phys. Rev. D 21, 2308 (1980).

[3] S. W. Otto and J.D. Stack, The SU(3) Heavy Quark Potential with High Statistics, Phys. Rev. Lett. 52, 2328 (1984).

[4] G. S. Bali, QCD forces and heavy quark bound states, Phys. Rep. 343, 1 (2001).

[5] E. Eichten, K. Gottfried, T. Kinoshita, J. B. Kogut, K. D. Lane, and T.-M. Yan, The Spectrum of Charmonium, Phys. Rev. Lett. 34, 369 (1975); 36, 1276(E) (1976).

[6] G. Aarts, Introductory lectures on lattice QCD at nonzero baryon number, J. Phys. Conf. Ser. 706, 022004 (2016).

[7] J. S. Schwinger, Gauge invariance and mass. 2., Phys. Rev. 128, 2425 (1962).

[8] S. R. Coleman, R. Jackiw, and L. Susskind, Charge shielding and quark confinement in the massive schwinger model, Ann. Phys. (N.Y.) 93, 267 (1975).

[9] D. J. Gross, I. R. Klebanov, A. V. Matytsin, and A. V. Smilga, Screening versus confinement in $(1+1)$-dimensions, Nucl. Phys. B461, 109 (1996).

[10] J. Preskill, Quantum computing in the NISQ era and beyond, Quantum 2, 79 (2018).

[11] E. A. Martinez et al., Real-time dynamics of lattice gauge theories with a few-qubit quantum computer, Nature (London) 534, 516 (2016).

[12] C. Muschik, M. Heyl, E. Martinez, T. Monz, P. Schindler, B. Vogell, M. Dalmonte, P. Hauke, R. Blatt, and P. Zoller, U(1) Wilson lattice gauge theories in digital quantum simulators, New J. Phys. 19, 103020 (2017).

[13] N. Klco, E. F. Dumitrescu, A. J. McCaskey, T. D. Morris, R. C. Pooser, M. Sanz, E. Solano, P. Lougovski, and M. J. Savage, Quantum-classical computation of Schwinger model dynamics using quantum computers, Phys. Rev. A 98, 032331 (2018).

[14] C. Kokail et al., Self-verifying variational quantum simulation of lattice models, Nature (London) 569, 355 (2019).

[15] G. Magnifico, M. Dalmonte, P. Facchi, S. Pascazio, F. V. Pepe, and E. Ercolessi, Real time dynamics and confinement in the $\mathbb{Z}_{n}$ Schwinger-Weyl lattice model for $1+1$ QED, Quantum 4, 281 (2020).

[16] B. Chakraborty, M. Honda, T. Izubuchi, Y. Kikuchi, and A. Tomiya, Digital quantum simulation of the Schwinger model with topological term via adiabatic state preparation, arXiv:2001.00485.

[17] A. Yamamoto, Quantum variational approach to lattice gauge theory at nonzero density, Phys. Rev. D 104, 014506 (2021).

[18] D. E. Kharzeev and Y. Kikuchi, Real-time chiral dynamics from a digital quantum simulation, Phys. Rev. Research 2, 023342 (2020).

[19] W. A. de Jong, K. Lee, J. Mulligan, M. Płoskoń, F. Ringer, and X. Yao, Quantum simulation of non-equilibrium dynamics and thermalization in the Schwinger model, arXiv:2106.08394.

[20] H. Bernien et al., Probing many-body dynamics on a 51atom quantum simulator, Nature (London) 551, 579 (2017).

[21] F. M. Surace, P. P. Mazza, G. Giudici, A. Lerose, A. Gambassi, and M. Dalmonte, Lattice Gauge Theories and
String Dynamics in Rydberg Atom Quantum Simulators, Phys. Rev. X 10, 021041 (2020).

[22] S. P. Jordan, K. S. M. Lee, and J. Preskill, Quantum algorithms for quantum field theories, Science 336, 1130 (2012).

[23] S. P. Jordan, K. S. M. Lee, and J. Preskill, Quantum computation of scattering in scalar quantum field theories, Quantum Inf. Comput. 14, 1014 (2014).

[24] S. P. Jordan, K. S. M. Lee, and J. Preskill, Quantum algorithms for fermionic quantum field theories, arXiv:1404.7115.

[25] L. Garcia-Alvarez, J. Casanova, A. Mezzacapo, I. L. Egusquiza, L. Lamata, G. Romero, and E. Solano, FermionFermion Scattering in Quantum Field Theory with Superconducting Circuits, Phys. Rev. Lett. 114, 070502 (2015).

[26] U.-J. Wiese, Towards quantum simulating QCD, Nucl. Phys. A931, 246 (2014).

[27] D. Marcos, P. Widmer, E. Rico, M. Hafezi, P. Rabl, U. J. Wiese, and P. Zoller, Two-dimensional lattice gauge theories with superconducting quantum circuits, Ann. Phys. (Amsterdam) 351, 634 (2014).

[28] A. Mezzacapo, E. Rico, C. Sabin, I. L. Egusquiza, L. Lamata, and E. Solano, Non-Abelian $S U(2)$ Lattice Gauge Theories in Superconducting Circuits, Phys. Rev. Lett. 115, 240502 (2015).

[29] A. Macridin, P. Spentzouris, J. Amundson, and R. Harnik, Electron-Phonon Systems on a Universal Quantum Computer, Phys. Rev. Lett. 121, 110504 (2018).

[30] H. Lamm and S. Lawrence, Simulation of Nonequilibrium Dynamics on a Quantum Computer, Phys. Rev. Lett. 121, 170501 (2018).

[31] N. Klco and M. J. Savage, Digitization of scalar fields for quantum computing, Phys. Rev. A 99, 052335 (2019).

[32] E. Gustafson, Y. Meurice, and J. Unmuth-Yockey, Quantum simulation of scattering in the quantum Ising model, Phys. Rev. D 99, 094503 (2019).

[33] , A. Alexandru, P. F. Bedaque, H. Lamm, and S. Lawrence (NuQS Collaboration), $\sigma$ Models on Quantum Computers, Phys. Rev. Lett. 123, 090501 (2019).

[34] N. Klco and M. J. Savage, Minimally-entangled state preparation of localized wavefunctions on quantum computers, Phys. Rev. A 102, 012612 (2020).

[35] N. Klco, J. R. Stryker, and M. J. Savage, SU(2) non-Abelian gauge field theory in one dimension on digital quantum computers, Phys. Rev. D 101, 074512 (2020).

[36] H. Lamm, S. Lawrence, and Y. Yamauchi (NuQS Collaboration), Parton physics on a quantum computer, Phys. Rev. Research 2, 013272 (2020).

[37] N. Mueller, A. Tarasov, and R. Venugopalan, Deeply inelastic scattering structure functions on a hybrid quantum computer, Phys. Rev. D 102, 016007 (2020).

[38] E. Gustafson, P. Dreher, Z. Hang, and Y. Meurice, Real time evolution of a one-dimensional field theory on a 20 qubit machine, Quantum Sci. Technol. 6, 045020 (2021).

[39] A. J. Buser, T. Bhattacharya, L. Cincio, and R. Gupta, State preparation and measurement in a quantum simulation of the $\mathrm{O}(3)$ sigma model, Phys. Rev. D 102, 114514 (2020).

[40] F. Mei, Q. Guo, Y.-F. Yu, L. Xiao, S.-L. Zhu, and S. Jia, Digital Simulation of Topological Matter on Programmable Quantum Processors, Phys. Rev. Lett. 125, 160503 (2020). 
[41] F. Arute et al., Observation of separated dynamics of charge and spin in the Fermi-Hubbard model, arXiv:2010.07965.

[42] A. J. Buser, H. Gharibyan, M. Hanada, M. Honda, and J. Liu, Quantum simulation of gauge theory via orbifold lattice, J. High Energy Phys. 09 (2021) 034.

[43] N. Klco and M. J. Savage, Fixed-point quantum circuits for quantum field theories, Phys. Rev. A 102, 052422 (2020).

[44] J. Liu and Y. Xin, Quantum simulation of quantum field theories as quantum chemistry, J. High Energy Phys. 12 (2020) 011.

[45] Y. Su, H.-Y. Huang, and E. T. Campbell, Nearly tight Trotterization of interacting electrons, Quantum 5, 495 (2021).

[46] E. J. Gustafson and H. Lamm, Toward quantum simulations of $\mathrm{Z}_{2}$ gauge theory without state preparation, Phys. Rev. D 103, 054507 (2021).

[47] J. Barata, N. Mueller, A. Tarasov, and R. Venugopalan, Single-particle digitization strategy for quantum computation of a $\phi^{4}$ scalar field theory, Phys. Rev. A 103, 042410 (2021).

[48] A. Ciavarella, N. Klco, and M. J. Savage, Trailhead for quantum simulation of SU(3) Yang-Mills lattice gauge theory in the local multiplet basis, Phys. Rev. D 103, 094501 (2021).

[49] H. Gharibyan, M. Hanada, M. Honda, and J. Liu, Toward simulating superstring/M-theory on a quantum computer, J. High Energy Phys. 07 (2021) 140.

[50] M. G. Echevarria, I. L. Egusquiza, E. Rico, and G. Schnell, Quantum simulation of light-front parton correlators, Phys. Rev. D 104, 014512 (2021).

[51] D. Paulson, L. Dellantonio, J. F. Haase, A. Celi, A. Kan, A. Jena, C. Kokail, R. van Bijnen, K. Jansen, P. Zoller, and C. A. Muschik, Simulating 2D effects in lattice gauge theories on a quantum computer, PRX Quantum 2, 030334 (2021).

[52] J. Liu and Y.-Z. Li, Quantum simulation of cosmic inflation, Phys. Rev. D 104, 086013 (2021).

[53] Z. Davoudi, I. Raychowdhury, and A. Shaw, Search for efficient formulations for Hamiltonian simulation of nonAbelian lattice gauge theories, Phys. Rev. D 104, 074505 (2021).

[54] C. W. Bauer, B. Nachman, and M. Freytsis, Simulating Collider Physics on Quantum Computers Using Effective Field Theories, Phys. Rev. Lett. 127, 212001 (2021).

[55] E. Zohar, J. I. Cirac, and B. Reznik, Simulating Compact Quantum Electrodynamics with Ultracold Atoms: Probing Confinement and Nonperturbative Effects, Phys. Rev. Lett. 109, 125302 (2012).

[56] D. Banerjee, M. Dalmonte, M. Muller, E. Rico, P. Stebler, U. J. Wiese, and P. Zoller, Atomic Quantum Simulation of Dynamical Gauge Fields coupled to Fermionic Matter: From String Breaking to Evolution after a Quench, Phys. Rev. Lett. 109, 175302 (2012).

[57] E. Zohar, J. I. Cirac, and B. Reznik, Cold-Atom Quantum Simulator for SU(2) Yang-Mills Lattice Gauge Theory, Phys. Rev. Lett. 110, 125304 (2013).

[58] D. Banerjee, M. Bogli, M. Dalmonte, E. Rico, P. Stebler, U. J. Wiese, and P. Zoller, Atomic Quantum Simulation of U (N) and SU(N) Non-Abelian Lattice Gauge Theories, Phys. Rev. Lett. 110, 125303 (2013).
[59] U.-J. Wiese, Ultracold quantum gases and lattice systems: Quantum simulation of lattice gauge theories, Ann. Phys. (Amsterdam) 525, 777 (2013).

[60] E. Zohar, J. I. Cirac, and B. Reznik, Quantum simulations of lattice gauge theories using ultracold atoms in optical lattices, Rep. Prog. Phys. 79, 014401 (2016).

[61] A. Bazavov, Y. Meurice, S.-W. Tsai, J. Unmuth-Yockey, and J. Zhang, Gauge-invariant implementation of the Abelian Higgs model on optical lattices, Phys. Rev. D 92, 076003 (2015).

[62] E. Zohar, A. Farace, B. Reznik, and J. I. Cirac, Digital lattice gauge theories, Phys. Rev. A 95, 023604 (2017).

[63] A. Bermudez, G. Aarts, and M. Muller, Quantum Sensors for the Generating Functional of Interacting Quantum Field Theories, Phys. Rev. X 7, 041012 (2017).

[64] T. V. Zache, F. Hebenstreit, F. Jendrzejewski, M. K. Oberthaler, J. Berges, and P. Hauke, Quantum simulation of lattice gauge theories using Wilson fermions, Sci. Technol. 3, 034010 (2018).

[65] J. Zhang, J. Unmuth-Yockey, J. Zeiher, A. Bazavov, S. W. Tsai, and Y. Meurice, Quantum Simulation of the Universal Features of the Polyakov Loop, Phys. Rev. Lett. 121, 223201 (2018).

[66] H.-H. Lu et al., Simulations of subatomic many-body physics on a quantum frequency processor, Phys. Rev. A 100, 012320 (2019).

[67] A. Roy, D. Schuricht, J. Hauschild, F. Pollmann, and H. Saleur, The quantum sine-Gordon model with quantum circuits, Nucl. Phys. B968, 115445 (2021).

[68] M. C. Tran, Y. Su, D. Carney, and J. M. Taylor, Faster digital quantum simulation by symmetry protection, PRX Quantum 2, 010323 (2021).

[69] I. Raychowdhury and J. R. Stryker, Loop, string, and hadron dynamics in SU(2) Hamiltonian lattice gauge theories, Phys. Rev. D 101, 114502 (2020).

[70] E. J. Gustafson, Prospects for simulating a qudit-based model of $(1+1) \mathrm{D}$ scalar QED, Phys. Rev. D 103, 114505 (2021).

[71] T. Banks, L. Susskind, and J. B. Kogut, Strong coupling calculations of lattice gauge theories: $(1+1)$-dimensional exercises, Phys. Rev. D 13, 1043 (1976).

[72] C. J. Hamer, W.-H. Zheng, and J. Oitmaa, Series expansions for the massive Schwinger model in Hamiltonian lattice theory, Phys. Rev. D 56, 55 (1997).

[73] J. B. Kogut and L. Susskind, Hamiltonian formulation of Wilson's lattice gauge theories, Phys. Rev. D 11, 395 (1975).

[74] P. Jordan and E. Wigner, Über das paulische äquivalenzverbot, Z. Phys. 47, 631 (1928).

[75] E. Farhi, J. Goldstone, S. Gutmann, and M. Sipser, Quantum computation by adiabatic evolution, arXiv:quant-ph/ 0001106.

[76] S. Jansen, M.-B. Ruskai, and R. Seiler, Bounds for the adiabatic approximation with applications to quantum computation, J. Math. Phys. (N.Y.) 48, 102111 (2007).

[77] S. Lloyd, Universal quantum simulators, Science 273, 1073 (1996).

[78] M. Suzuki, General theory of fractal path integrals with applications to many-body theories and statistical physics, J. Math. Phys. (N.Y.) 32, 400 (1991). 
[79] S. R. Coleman, More about the massive Schwinger model, Ann. Phys. (N.Y.) 101, 239 (1976).

[80] T. Byrnes, P. Sriganesh, R. Bursill, and C. Hamer, Density matrix renormalization group approach to the massive Schwinger model, Phys. Rev. D 66, 013002 (2002).

[81] S. Iso and H. Murayama, Hamiltonian formulation of the Schwinger model: Nonconfinement and screening of the charge, Prog. Theor. Phys. 84, 142 (1990).

[82] M. A. Nielsen and I. L. Chuang, Quantum Computation and Quantum Information: 10th Anniversary Edition (Cambridge University Press, Cambridge, England, 2011).

[83] B. Buyens, J. Haegeman, H. Verschelde, F. Verstraete, and K. Van Acoleyen, Confinement and String Breaking for $\mathrm{QED}_{2}$ in the Hamiltonian Picture, Phys. Rev. X 6, 041040 (2016).

[84] J. B. Kogut, D. K. Sinclair, R. B. Pearson, J. L. Richardson, and J. Shigemitsu, The fluctuating string of lattice gauge theory: The heavy quark potential, the restoration of rotational symmetry and roughening, Phys. Rev. D 23, 2945 (1981).

[85] R. B. Pearson and J. L. Richardson, The quark - anti-quark state in the strong coupling limit of lattice QCD, Phys. Rev. D 25, 2658 (1982).
[86] N. Wiebe and N.S. Babcock, Improved error-scaling for adiabatic quantum evolutions, New J. Phys. 14, 013024 (2012).

[87] A. F. Shaw, P. Lougovski, J. R. Stryker, and N. Wiebe, Quantum algorithms for simulating the lattice Schwinger model, Quantum 4, 306 (2020).

[88] B. Eastin and E. Knill, Restrictions on Transversal Encoded Quantum Gate Sets, Phys. Rev. Lett. 102, 110502 (2009).

[89] S. Bravyi and J. Haah, Magic-state distillation with low overhead, Phys. Rev. A 86, 052329 (2012).

[90] A. Paetznick and K. M. Svore, Repeat-until-success: Nondeterministic decomposition of single-qubit unitaries, Quantum Inf. Comput. 14, 1277 (2014).

[91] A. Bocharov, M. Roetteler, and K. M. Svore, Efficient Synthesis of Universal Repeat-Until-Success Quantum Circuits, Phys. Rev. Lett. 114, 080502 (2015).

[92] M. C. Tran, Y. Su, D. Carney, and J. M. Taylor, Faster digital quantum simulation by symmetry protection, PRX Quantum 2, 010323 (2021).

[93] C. Adam, Massive Schwinger model within mass perturbation theory, Ann. Phys. (N.Y.) 259, 1 (1997).

[94] T. Okuda (to be published).

[95] L. Funcke, K. Jansen, and S. Kühn, Topological vacuum structure of the Schwinger model with matrix product states, Phys. Rev. D 101, 054507 (2020). 